\title{
Control Systems Analysis for the Fornasini-Marchesini 2D Systems Model - Progress after Four Decades
}

\author{
ARTICLE HISTORY \\ Compiled October 2, 2018

\begin{abstract}
The development of a control systems theory for multidimensional linear systems, i.e., systems with more than one independent variable is an area where a very significant volume of research is based on representations for the underlying dynamics introduced in the $1970 \mathrm{~s}$. One of these is the Fornasini-Marchesini state-space model published in 1978. This paper reviews the significant developments in the succeeding four decades in both theory and application, where the second area includes examples where this model description is an enabler the analysis of other classes of systems.
\end{abstract}

\section{KEYWORDS}

Fornasini-Marchesini models, Roesser model, linear repetitive process model, control theoretic analysis, control design.

\section{Introduction}

A very substantial part of the literature on control theory and its applications addresses systems governed by ordinary differential equations or difference equations in the discrete case, where the latter may result from sampling the former. One starting point for the development of multidimensional, also termed $n \mathrm{D}$, systems control theory was systems described by partial differential or difference equations. In these systems, the independent variables may represent different space coordinates, with examples in image processing applications, or mixed time and space variables as in processing seismic data. Multidimensional models also arise in the analysis of systems described by particular types of functional differential equations in one independent variable, such as delay-differential systems.

As for standard systems, i.e., the single independent variable is time, also termed $1 \mathrm{D}$ in some of the literature, the starting point in much of the literature for the analysis of $n \mathrm{D}$ linear systems is state-space models and transfer-function descriptions of the dynamics. The major state-space model classes considered are due, respectively, to Roesser (1975) and Fornasini and Marchesini (1978). In the former case, the state vector is partitioned into a sub-vector for each direction of information propagation whereas in the latter a single state vector is used.

Repetitive processes make a series of sweeps, termed passes, through a set of dynamics defined over a finite duration known as the pass length. On completion of a pass, the process resets to the starting location and the next pass can begin, either immediately after the resetting operation is complete or after a further period of time has elapsed. The output on each pass is termed the pass profile and acts as a forcing 
function on the next pass profile and thereby contributes to its dynamics. This interpass interaction can result in oscillations that increase in amplitude from pass-to-pass and which cannot be removed by $1 \mathrm{D}$ control action. Repetitive process dynamics occur in the modeling and control of industrial examples, such as Rogers, Galkowski, and Owens (2007), which also references the original modeling work.

These three representations of $2 \mathrm{D} / n \mathrm{D}$ systems have common structural properties and this raises the question: is it possible to develop a unified setting for control systems analysis and control law design? This question has been a recurring theme in research over the years and for linear dynamics substantial progress has been reported. For nonlinear dynamics, however, this route does not offer a way forward (unless the dynamics can be locally approximated by a linear model).

The solution of $n \mathrm{D}$ systems and control design problems require a mathematical setting to address problems whose formulation and solution, for linear dynamics, can involve the use of functions and polynomials in more than one complex or real variables, where fundamental differences with the 1D linear systems case immediately arise. For example, transfer-function descriptions of the dynamics of linear time invariant systems releases a wealth of results from the theory of polynomials in one indeterminate for use in analysis and design, e.g., coprimeness and Bezout identities. In the $n \mathrm{D}$ case, coprimeness is, see also below, no longer a single concept and hence the polynomial approach in the $n \mathrm{D}$ case is much more complicated.

Four decades have elapsed since the Fornasini-Marchesini state-space model was published and the general aim of this paper is to provide a review of the critical developments in control systems analysis for this class of models. The coverage is, except for one area, for $2 \mathrm{D}$ systems but there are differences with $n \mathrm{D}, n>2$, case for which coprimeness again provides an example. In the 1D systems case, zero, factor and minor coprimeness are the same property, for 2D systems, minor and factor coprimeness are equivalent and zero coprimeness is a different property. For $n \geq 3$ none of these coprimeness properties are equivalent but it can be shown that zero coprimeness implies minor coprimeness, which, in turn, implies factor coprimeness, see, e.g., Youla and Gnavi (1979) for a detailed treatment of this area. The next section considers the modeling of such systems.

In this paper, the zero and identity matrices of compatible dimensions, respectively, are denoted by $I$ and 0 . Also $\rho(\cdot)$ denotes the spectral radius of its matrix argument, $\succ 0$ a symmetric positive-definite matrix, $\prec 0$ a symmetric negative definite matrix and $\otimes$ denotes the matrix Kronecher product.

\section{Models for 2D Dynamics}

\subsection{Discrete Dynamics}

The first significant developments in 2D linear systems theory arose in the 1970s with the introduction of two state-space models, the first of which is the Roesser (1975) model motivated by image processing problems, where there are several ways of modeling the dynamics, e.g., convolutional sums, partial difference, or recursive, equations and transfer-functions. In Roesser (1975) it is argued that a state-space model description is a powerful way to study $2 \mathrm{D}$ systems theoretical and design problems. The aim was to develop a state-space model for 2D systems, which was subsequently generalized to the $n \mathrm{D}$ case, $n>2$.

In the introduction to Roesser (1975) it is stated that 'Temporal systems have are 
inherently non-anticipatory and are often treated as such for the sake of physicalrealization in real time; whereas spatial systems do not have causality as an inherent limitation.' This means that for 2D spatio-temporal systems, as one example, an image processor may have both left-to-right and right-to-left dependency. In a temporal system time enforces a natural partition of past present and future, i.e., causality. Moreover, in Roesser (1975) the same form of causality was assumed, with the aim of establishing a close parallel with a spatial model, even though causality is not necessary for physical realizability in real space. In the absence of causality, the processor is termed bilateral.

The Roesser state-space model for discrete linear systems recursive over the upper right quadrant of the $2 \mathrm{D}$ plane, i.e., $\mathcal{D}_{u p}\{(i, j), i \geq 0, j \geq 0\}$ has the form

$$
\begin{aligned}
{\left[\begin{array}{c}
x^{h}(i+1, j) \\
x^{v}(i, j+1)
\end{array}\right] } & =\left[\begin{array}{ll}
A_{11} & A_{12} \\
A_{21} & A_{22}
\end{array}\right]\left[\begin{array}{l}
x^{h}(i, j) \\
x^{v}(i, j)
\end{array}\right]+\left[\begin{array}{l}
B_{1} \\
B_{2}
\end{array}\right] u(i, j) \\
y(i, j) & =\left[\begin{array}{ll}
C_{1} & C_{2}
\end{array}\right]\left[\begin{array}{l}
x^{h}(i, j) \\
x^{v}(i, j)
\end{array}\right]+D u(i, j) .
\end{aligned}
$$

In this model the state vector consists of sub-vectors for the vertical and horizontal axes, i.e., $x^{h}(i, j) \in \mathbb{R}^{d_{1}}$ and $x^{v}(i, j) \in \mathbb{R}^{d_{2}}$, respectively, where these vectors are also termed the horizontal and vertical sub-vectors in much of the literature. The output vector $y(i, j) \in \mathbb{R}^{q}$ and the input vector $u(i, j) \in \mathbb{R}^{l}$ complete with model structure together with the boundary conditions $x^{h}(0, j)=f_{1}(j), j \geq 0$ and $x^{v}(i, 0)=f_{2}(j), i \geq$ 0 , where the $d_{1} \times 1$ vector $f_{1}(j)$ and the $d_{2} \times 1$ vector $f_{2}(i)$ have known entries.

The Fornasini-Marchesini state-space model Fornasini and Marchesini (1978) is an alternative representation for $2 \mathrm{D}$ systems over $\mathcal{D}_{u p}$. In fact, there are a family of such models and one of them is the first order with the structure

$$
\begin{aligned}
x(i+1, j+1) & =A_{1} x(i+1, j)+A_{2} x(i, j+1) \\
& +B_{1} u(i+1, j)+B_{2} u(i, j+1), \\
y(i, j) & =C x(i, j)+D u i, j),
\end{aligned}
$$

where the vectors $z \in \mathbb{R}^{n}, y \in \mathbb{R}^{q}$ and $u \in \mathbb{R}^{l}$ are the (appropriately dimensioned) state, output and input vectors, respectively, with the boundary conditions defined as $x(0, j)=f_{1}(j), j \geq 0$ and $x(i, 0)=d_{1}(i), i \geq 0$, where the $n \times 1$ vector $f_{1}(j)$ and the $n \times 1$ vector $d_{1}(i)$ have known entries.

A second order Fornasini-Marchesini state-space model has the structure

$$
\begin{aligned}
x(i+1, j+1) & =A_{1} x(i+1, j)+A_{2} x(i, j+1)+A_{3} x(i, j) \\
& +B_{3} u(i, j) \\
y(i, j) & =C x(i, j)+D u(i, j),
\end{aligned}
$$

where the vectors involved and the boundary conditions are as in (2). The particular case of this state-space model that results when $A_{3}=-A_{1} A_{2}$ is known the Attasi 


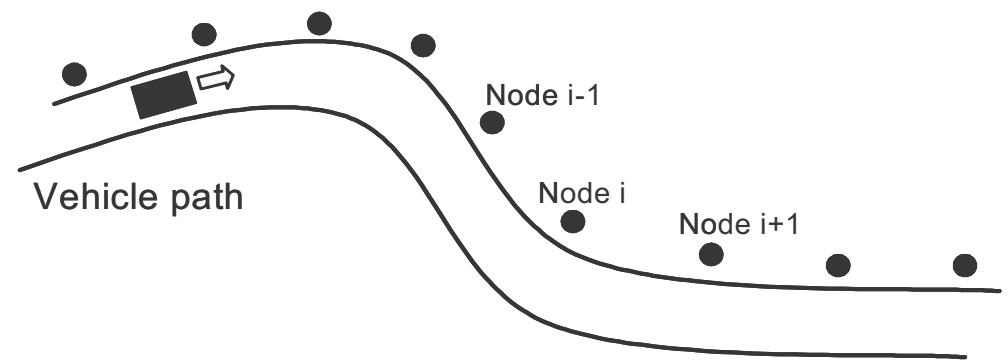

Figure 1. A vehicle path under surveillance. The path is equipped with regularly spaced sensor nodes, represented by the black circles, placed in a $1 \mathrm{D}$ array.

model Attasi (1973). Adding extra input terms gives the general model Kurek (1985))

$$
\begin{aligned}
x(i+1, j+1) & =A_{1} x(i+1, j)+A_{2} x(i, j+1)+A_{3} x(i, j), \\
& +B_{1} u(i+1, j)+B_{2} u(i, j+1)+B_{3} u(i, j), \\
y(i, j) & =C x(i, j)+D u(i, j) .
\end{aligned}
$$

As a physically motivated example, consider a regularly placed grid sensor network, such as a vehicle path under surveillance, equipped with regularly spaced sensor nodes placed in a 1D array as shown in Figure 1. In this figure, the regularly spaced sensor nodes are denoted by black circles and the sensor number is denoted by $i$. The sensor node signals are sampled in time for discrete processing and let $j$ denote the sample number, resulting in a $2 \mathrm{D}$ discrete spatio-temporal signal.

To describe the updating structure, consider sample instance $j$ at node $i$ and assume that the dynamics are linear. Then the sensor output, denoted by $y(i+1, j)$, is a linear combination of the state vector entries and the node generates the state vector at the next time instant, i.e., $x(i+1, j+1)$, by combining the current sample instance state vector at node $i+1$, i.e., $x(i+1, j)$, with the current sample instance state vector at the former node $i$, i.e., $x(i, j)$, and the input or control vector to the node at the current sample instance, i.e., $u\left(n_{1}+1, n_{2}\right)$. On completion of the computations, the node transmits its state information to the next node and so on.

The state updating dynamics are described by the sensor network model

$$
x(i+1, j+1)=A_{1} x(i+1, j)+A_{2} x(i, j)+B_{1} u(i+1, j) .
$$

Suppose also that $i$ and $j$ are restricted to nonnegative values. Then the dynamics described by (4) can be pictured as evolving over the positive quadrant of the $2 \mathrm{D}$ plane with axes $i$, and $j$, respectively, where each node is a point in the 2D plane.

Another class of 2D models is those where there is an implicit dependence between shifted state variables and the current counterparts. Such models represent a collection of dynamic and static dependencies and the resulting models are often termed descriptor or singular. The singular first order Fornasini-Marchesini model has the following state-space model where the matrix $E$ is singular

$$
\begin{aligned}
E z(i+1, j+1) & =A_{1} z(i+1, j)+A_{2} z(i, j+1) \\
& +B_{1} u(i+1, j)+B_{2} u(i, j+1), \\
y(i, j) & =C z(i, j)+D u(i, j) .
\end{aligned}
$$


The singular second order Fornasini-Marchesini state-space model is

$$
\begin{aligned}
E z(i+1, j+1) & =A_{1} z(i+1, j)+A_{2} z(i, j+1)+A_{3} z(i, j)+B_{3} u(i, j) \\
y(i, j) & =C z(i, j)+D u(i, j)
\end{aligned}
$$

Singular versions of the Roesser state-space model can also be defined.

Repetitive process are another class of $2 \mathrm{D}$ systems that are characterized by a series of sweeps, termed passes, through a set of dynamics defined over a finite duration known as the pass length. The state-space model for discrete linear processes has the form

$$
\begin{aligned}
x_{k+1}(p+1) & =\mathbb{A} x_{k+1}(p)+\mathbb{B} u_{k+1}(p)+\mathbb{B}_{0} y_{k}(p), \\
y_{k+1}(p) & =\mathbb{C} x_{k+1}(p)+\mathbb{D} u_{k+1}(p)+\mathbb{D}_{0} y_{k}(p),
\end{aligned}
$$

for $0 \leq p \leq \alpha-1$, where $\alpha<\infty$ denotes the number of samples along the pass ( $\alpha$ times the sampling period is equal to the pass length) and on pass $k, x_{k}(p) \in \mathbb{R}^{m_{1}}$ is the state vector, $y_{k}(p) \in \mathbb{R}^{m_{2}}$ is the pass profile vector, which serves also as the system output and $u_{k}(p) \in \mathbb{R}^{r}$ is the control input vector. Boundary conditions are the state initial vector for each pass and the pass profile on the initial pass, i.e., $k=0$. An obvious choice is

$$
x_{k+1}(0)=d_{k+1}, \quad k \geq 0, y_{0}(p)=f(p), \quad 0 \leq p \leq \alpha-1,
$$

where $f(p)$ is a given initial pass profile vector and the vector $d_{k+1}$ has known constant entries.

The structure of the boundary conditions for repetitive processes and especially the state initial vector on each pass is much more complex than for $1 \mathrm{D}$ systems. The boundary conditions of $(9)$ have a state initial vector $x_{k}(0)$ that is independent of the previous pass dynamics. A more general case is where the state initial vector sequence $x_{k+1}(0), k \geq 0$, is of the form

$$
x_{k+1}(0)=d_{k+1}+\sum_{j=0}^{\alpha-1} J_{j} y_{k}(j), k \geq 0,
$$

where $J_{j}, 1 \leq j \leq \alpha-1$, is an $n \times m$ matrix. In this case, the state initial vector on each pass is an explicit function of samples along the previous pass profile. Combined with the initial pass profile $y_{0}(p)$ of $(8)$ the result is termed dynamic boundary conditions.

Structural similarities exist between discrete linear repetitive processes and the Roesser and Fornasini-Marchesini state-space models. The question is: can these be made precise and hence the possibility that results developed in one area can be applied to the other and vice versa? The answer to this question is more complicated than it first appears. Figure 2 gives some examples of separation-sets for the Roesser model where the system dynamics evolve over a plane where $i$ is a spatial variable and $j$ a temporal variable and there can be no linear ordering on the plane and hence no time enforced separation into past, present and future. The separation set for $2 \mathrm{D}$ systems is a generalization of idea where past represents already computed or known values and future those to be computed by a recursive algorithm, starting from the values that lie on this set. 


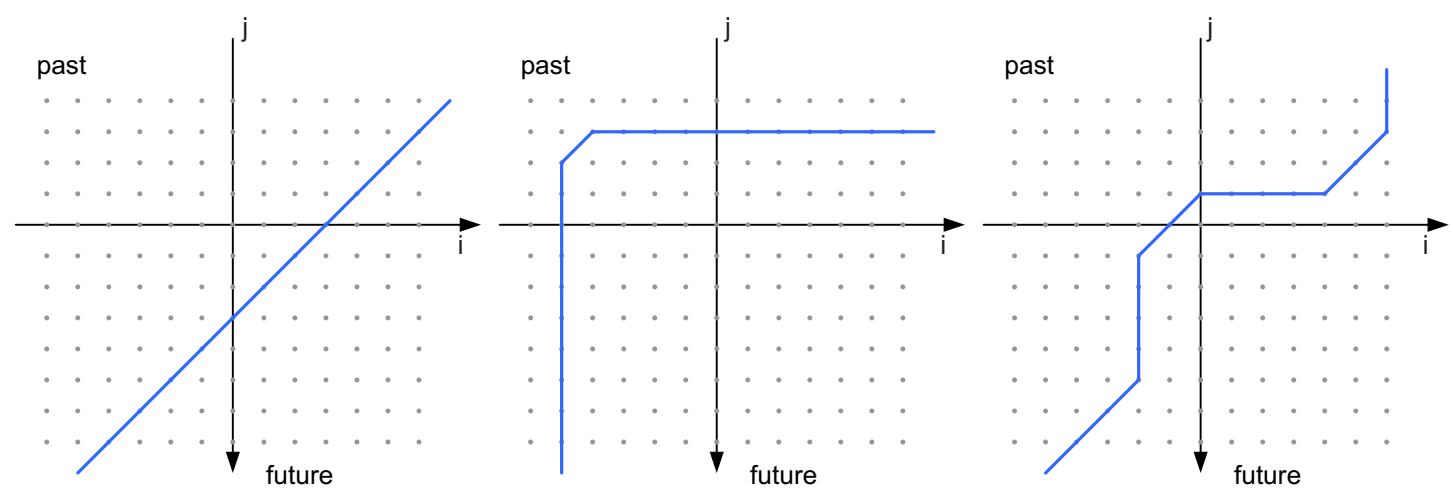

Figure 2. Separation sets for the Roesser and Fornasini-Marchesini state-space models. The blue lines represent these sets and the 2D system dynamics evolve over a plane, where $i$ is a spatial variable and $j$ a temporal variable. No linear ordering on the plane is possible and hence no time enforced separation into past, present, and future.

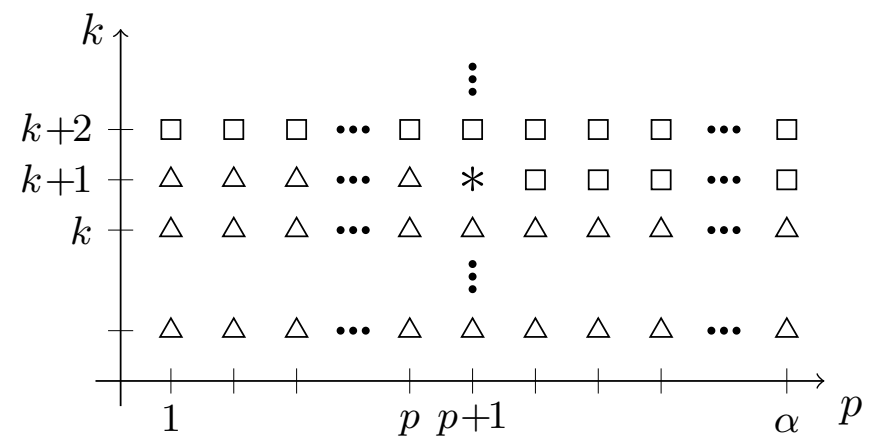

Figure 3. Evolution of the dynamics of a discrete repetitive process: $*$ - current point, $\triangle-$ past points, $\square-$ future points.

Repetitive process dynamics evolve over an infinite number of passes $k \geq 0$ and finite pass length $0 \leq p \leq \alpha-1, k \geq 0$. Unlike Roesser and Fornasini Marches models there is separation set partitioning past and future dynamics. For (discrete) repetitive processes at a given sample on the current pass the past consists of all previous passes together with those samples on the current pass already computed and the future consists of all sample instants to the end of the current pass and all future passes, see Figure 3. Also there are no Roesser or Fornasini-Marchesini equivalents of the dynamic boundary conditions of (10). However, as detailed later in this paper, in some cases it is possible to write the dynamics of discrete linear repetitive processes with boundary conditions of the form (9) as Roesser and Fornasini-Marchesini state-space models and vice versa and hence interchange systems theoretic concepts to mutual benefit. 


\subsection{Continuous-discrete dynamics}

Certain partial differential equations can be interpreted as 2D systems. The first order Fornasini-Marchesini state-space model has the form

$$
\begin{aligned}
\frac{\partial}{\partial t_{1}} \frac{\partial}{\partial t_{2}} z\left(t_{1}, t_{2}\right) & =A_{1} \frac{\partial}{\partial t_{1}} z\left(t_{1}, t_{2}\right)+A_{2} \frac{\partial}{\partial t_{2}} z\left(t_{1}, t_{2}\right) \\
& +B_{1} \frac{\partial}{\partial t_{1}} u\left(t_{1}, t_{2}\right)+B_{2} \frac{\partial}{\partial t_{2}} u\left(t_{1}, t_{2}\right), \\
y\left(t_{1}, t_{2}\right) & =C z\left(t_{1}, t_{2}\right)+D u\left(t_{1}, t_{2}\right),
\end{aligned}
$$

where $t_{1}, t_{2} \in \mathbb{R}$ and the vectors involved have identical definitions to those in (2). A second order Fornasini-Marchesini state-space model has the form

$$
\begin{aligned}
\frac{\partial}{\partial t_{1}} z\left(t_{1}, j+1\right) & =A_{1} \frac{\partial}{\partial t_{1}} z\left(t_{1}, j\right)+A_{2} z\left(t_{1}, j+1\right)+A_{3} z\left(t_{1}, j\right) \\
& +B_{3} u\left(t_{1}, j\right) \\
y\left(t_{1}, j\right) & =C z\left(t_{1}, j\right)+D u\left(t_{1}, j\right)
\end{aligned}
$$

with compatible boundary conditions. Both independent variables in the first of these last two models are continuous and in the second one variable is continuous and the other discrete. Other continuous-discrete models described by ordinary differential and discrete independent variables exist, e.g., differential linear repetitive processes where the dynamics along a pass are governed by an ordinary differential equation.

\subsection{Models for Robustness Analysis and Design}

If there is uncertainty associated with the dynamics of an example then a robustness analysis is required (unless the uncertainty can be ignored as not having a major influence on the response of the controlled system). As in the 1D systems case, the uncertainty is assumed to be modeled by one of two commonly used structures, termed, respectively, polytopic and norm-bounded. Polytopic uncertainty for a system described by (2) assumes that the matrices $A_{1}$ and $A_{2}$ in this model lie in the polytope

$$
\Psi=\left\{\left(A_{1}, A_{2}\right):\left(A_{1}, A_{2}\right)=\sum_{h=1}^{N} \beta_{h}\left(A_{1 h}, A_{2 h}\right), \sum_{h=1}^{N} \beta_{h}=1, \beta_{h} \geq 0,\right.
$$

where $N$ denotes the number of vertices.

Norm-bounded uncertainty is described by the state-space model

$$
\begin{aligned}
x(i, j+1) & =A_{1 \Delta} x(i+1, j)+A_{2 \Delta} x(i, j+1)+B_{1 \Delta} w(i, j+1) \\
& +B_{2 \Delta} w(i, j+1) \\
y(i, j) & =C x(i, j)+D w(i, j)
\end{aligned}
$$

where in comparison to $(2), w(i, j) \in \mathbb{R}^{q}$ represents an exogenous input (or disturbance). Also it is assumed that

$$
A_{1 \Delta}=A_{1}+\Delta A_{1}, A_{2 \Delta}=A_{2}+\Delta A_{2}, B_{1 \Delta}=B_{1}+\Delta B_{1}, B_{2 \Delta}=B_{2}+\Delta B_{2},
$$


where $\Delta A_{1}, \Delta A_{2}, \Delta B_{1}$ and Delta $B_{2}$ are matrices defining the norm-bounded parameter uncertainties and are assumed to satisfy

$$
\left[\begin{array}{llll}
\Delta A_{1} & \Delta A_{2} & \Delta B_{1} & \Delta B_{2}
\end{array}\right]=M F\left[\begin{array}{llll}
N_{A 1} & N_{A 2} & N_{B 1} & N_{B 2}
\end{array}\right],
$$

where $F \in \mathbb{R}^{q \times l}$ is an unknown real matrix that satisfies

$$
F F^{T} \preceq I
$$

and $M, N_{A 1}, N_{A 2}, N_{B 1}$ and $N_{B 2}$ are known matrices with real constant entries and compatible dimensions.

Note 1. In much of the $n \mathrm{D}$ systems literature the definition of a shift operator is different to the $1 \mathrm{D}$ systems case. In particular, for $2 \mathrm{D}$ systems let $z_{1}$ denote the shift operator for the $i$ direction and $z_{2}$ that for the $j$ direction. Then the action of $z_{1}$ and $z_{2}$, respectively, on, say $x(i, j)$, is defined as $z_{1} x(i+1, j)=x(i, j)$ and $z_{2} x(i, j+1)=$ $z_{2} x(i, j)$, i.e., a backward shift. In this paper the corresponding forward shifts are denoted, respectively, by $\hat{z}_{1}$ and $\hat{z}_{2}$.

\subsection{Behavioral systems models}

In the behavioral systems setting for the representation and analysis of linear systems the central object of study is the behavior $\mathcal{B}$, i.e., the set of trajectories that satisfy the laws of the system, the properties of $\mathcal{B}$, and how such properties are reflected in properties of the representations of $\mathcal{B}$.

In the behavioral approach Polderman and Willems (1998); Willems (1991) a dynamic system is a triple $\Sigma=(\mathbb{T}, \mathbb{W}, \mathcal{B})$, where $\mathbb{T}$ is the index set, $\mathbb{N}^{n}$ or $\mathbb{Z}^{n}$ in the case of $n \mathrm{D}$ systems, $\mathbb{W}$ is the signal space, usually $\mathbb{R}^{q}$, and $\mathcal{B}$ is the behavior, i.e., a family of trajectories evolving over $\mathbb{T}$ and taking their values in $\mathbb{W}$, which satisfy the dynamical laws of the system.

A Fornasini-Marchesini model in the behavioral setting is interpreted as a 6 tuple $\left(Z, X, E, F_{1}, F_{2}, F_{0}\right)$ where $Z$ and $X$ are finite-dimensional linear spaces and $E, F_{1}, F_{2}, F_{0}: Z \rightarrow X$ are linear maps such that

$$
\operatorname{rank}\left(\hat{z}_{1} \hat{z}_{2} E-\hat{z}_{1} F_{1}-\hat{z}_{2} F_{2}-F_{0}\right)=\operatorname{dim} X,
$$

or, equivalently, this matrix has full row rank over the ring $\mathbb{F}\left(s_{1}, s_{2}\right)$ of polynomials in two indeterminates. The spaces $X$ and $Z$ are, respectively, termed the space of states and the internal variable space.

Suppose that the map $E: Z \rightarrow X$ is surjective (maps onto all of $X$ ) and write $Z=X \oplus \mathbb{F}^{m}$ where $\oplus$ denotes the direct sum and $\mathbb{F}$ is identified with the kernel of this last map. Then for the model (4)

$$
E=(I, 0), F_{1}=(I, 0), F_{1}=\left(A_{1}, B_{1}\right), F_{2}=\left(A_{2}, B_{2}\right), F_{0}=\left(A_{0}, B_{0}\right) .
$$

A Fornasini-Marchesini model can therefore be viewed as an autoregressive model and the trajectories on any upper-right quadrant plane are defined using the equation

$$
E x(i+1, j+1)=F_{1} x(i+1, j)+F_{2} x(i, j+1)+F_{0} x(i, j) .
$$


The behavioral approach to analysis is the subject of Section 6 .

\section{Links between $2 \mathrm{D}$ systems models}

The Fornasini-Marchesini and Roesser models are not completely independent of each other. In particular, introducing the substitution

$$
\xi(i, j)=x(i, j+1)-A_{1} x(i, j)
$$

into the second order Fornasini-Marchesini state-space model (3) results in the following Roesser type model

$$
\begin{aligned}
{\left[\begin{array}{c}
\xi(i+1, j) \\
x(i, j+1)
\end{array}\right] } & =\left[\begin{array}{cc}
A_{2} & A_{3}+A_{2} A_{1} \\
I & A_{1}
\end{array}\right]\left[\begin{array}{l}
\xi(i, j) \\
z(i, j)
\end{array}\right]+\left[\begin{array}{c}
B \\
0
\end{array}\right] u(i, j), \\
y(i, j) & =\left[\begin{array}{ll}
0 & C
\end{array}\right]\left[\begin{array}{l}
\xi(i, j) \\
z(i, j)
\end{array}\right]+D u(i, j) .
\end{aligned}
$$

This equivalence requires, however, that the resulting Roesser model has the same dimensions for both the vertical and horizontal state subvectors. Also the first order Fornasini-Marchesini model is obtained from (2) on setting

$$
\begin{aligned}
& A_{1}=\left[\begin{array}{cc}
(*) & (*) \\
0 & 0
\end{array}\right], \quad A_{2}=\left[\begin{array}{cc}
0 & 0 \\
(*) & (*)
\end{array}\right], \\
& B_{1}=\left[\begin{array}{c}
(*) \\
0
\end{array}\right], \quad B_{2}=\left[\begin{array}{c}
0 \\
(*)
\end{array}\right],
\end{aligned}
$$

where $(*)$ denotes an arbitrary matrix of compatible dimensions.

Links also exist in the singular dynamics case. Introducing, as one example, the following substitution into the singular second order Fornasini-Marchesini state-space (7)

$$
\zeta(i, j)=\operatorname{Ex}(i, j+1)-A_{1} x(i, j)
$$

gives the Roesser state-space model

$$
\begin{aligned}
{\left[\begin{array}{cc}
I & -A_{2} \\
0 & E
\end{array}\right]\left[\begin{array}{c}
\zeta(i+1, j) \\
x(i, j+1)
\end{array}\right] } & =\left[\begin{array}{ll}
0 & A_{3} \\
I & A_{1}
\end{array}\right]\left[\begin{array}{l}
\zeta(i, j) \\
x(i, j)
\end{array}\right]+\left[\begin{array}{c}
B \\
0
\end{array}\right] u(i, j) \\
y(i, j) & =\left[\begin{array}{ll}
0 & C
\end{array}\right]\left[\begin{array}{l}
\zeta(i, j) \\
x(i, j)
\end{array}\right]+D u(i, j)
\end{aligned}
$$

The singular Fornasini-Marchesini state-space model can be used in the analysis of discrete linear repetitive processes described by (8) as detailed in Gałkowski, Rogers, and Owens (1995). Introduce the augmented state vector for (8) as

$$
z(k, p)=\left[x_{k}^{T}(p) y_{k}^{T}(p)\right]^{T} .
$$

Then the discrete linear repetitive process state-space model can be rewritten in the 
form

$$
\begin{aligned}
\widehat{E} z(k+1, p+1) & =\widehat{A}_{1} z(k+1, p)+\widehat{A}_{3} z(k, p)+\widehat{B}_{1} u(k+1, p), \\
y(k, p) & =\widehat{C} z(k, p)+\widehat{D} u(k, p),
\end{aligned}
$$

where

$$
\begin{aligned}
\widehat{E} & =\left[\begin{array}{ll}
I & 0 \\
0 & 0
\end{array}\right], \quad \widehat{A}_{1}=\left[\begin{array}{cc}
\mathbb{A} & 0 \\
\mathbb{C} & -I
\end{array}\right], \\
\widehat{A}_{3} & =\left[\begin{array}{ll}
0 & \mathbb{B}_{0} \\
0 & \mathbb{D}_{0}
\end{array}\right], \quad \widehat{B}_{1}=\left[\begin{array}{l}
\mathbb{B} \\
\mathbb{D}
\end{array}\right],
\end{aligned}
$$

which is a particular case of (4) with $\widehat{A}_{2}=0, \widehat{B}_{2}=\widehat{B}_{3}=0$, where to make these models distinct the matrices $A_{i}, B_{i}, \quad i=1,2,3, C, D$ have been replaced by $\widehat{A}_{i}, \widehat{B}_{i}, \quad i=1,2,3, \widehat{C}, \widehat{D}$.

\subsection{Nonlinear dynamics}

The 2D discrete nonlinear systems considered in this section are described by the Fornasini-Marchesini state-space model

$$
x_{i+1, j+1}=f\left(x_{i, j+1}, x_{i+1, j}, u_{i, j+1}, u_{i+1, j}\right), i \geq 0, j \geq 0,
$$

where $x_{i, j} \in \mathbb{R}^{n_{x}}$ is the local state vector, $u_{i, j} \in \mathbb{R}^{n_{u}}$ is the input vector, and $f$ is a vector-valued function whose entries are assumed to be such that $f(0,0,0,0)=0$ and hence an equilibrium at the origin. The boundary conditions are assumed to be of the form

$$
x_{i, 0}=\xi_{0}(i), i \geq 0, x_{0, j}=\eta_{0}(j), j \geq 0,(i, j) \neq(0,0),
$$

where $\xi(i)$ and $\eta(j)$ are vectors whose entries, respectively, are known functions of $i$ and $j$.

\section{Stability and structural properties}

The stability of 2D linear systems has a long history with many contributions, where Huang (1972); Justice and Shanks (1973) are among the first for the inputoutput description of the dynamics, i.e., a bounded input produces a bounded output over the domain of operation, where boundedness is interpreted in terms of the norm on the underlying function space. This form of stability for the Fornasini-Marchesini model is discussed again later in the section. As in the 1D systems case, internal, or state, stability, is also of interest. Let $\|\cdot\|$ denote the Euclidean norm on $\mathbb{R}^{n}$ and introduce

$$
\mathcal{X}_{r}=\left\{x(i, j): x(i, j) \in \mathbb{R}^{n}, i+j=r\right\}
$$


and also

$$
\left\|\mathcal{X}_{r}\right\|=\sup _{n \in \mathbb{Z}}\|x(r-n, n)\|
$$

Then the definition of asymptotic stability for processes described by (5) in Fornasini and Marchesini (1978) is as follows.

Definition 4.1. A 2D discrete linear system is asymptotically stable, assuming $u=0$, if for finite $\left\|\mathcal{X}_{0}\right\|$

$$
\left\|\mathcal{X}_{k}\right\| \rightarrow 0, k \rightarrow \infty
$$

In physical terms, for the autonomous system the response to bounded initial conditions decays to zero as $k \rightarrow \infty$.

The stability of 1D linear systems can be characterized through the characteristic polynomial in one indeterminate and for $2 \mathrm{D}$ systems described by (5), the natural generalization is the following polynomial in two indeterminates

$$
\rho\left(z_{1}, z_{2}\right)=\operatorname{det}\left(I-z_{1} A_{1}-z_{2} A_{2}\right)
$$

and the following result is proved as Proposition 3 in Fornasini and Marchesini (1978).

Theorem 4.2. A $2 D$ discrete linear system described by (5) is asymptotically stable in the sense of Definition 4.1 if and only if

$$
\rho\left(z_{1}, z_{2}\right) \neq 0 \text { in } \mathcal{D}
$$

where

$$
\mathcal{D}=\left\{\left(z_{1}, z_{2}\right) \in \mathbb{C} \times \mathbb{C}:\left|z_{1}\right| \leq 1,\left|z_{2}\right| \leq 1\right\}
$$

Testing (31) is not feasible except a few trivial cases and this prompted a significant volume of work on efficient stability tests in the polynomial setting and later, via Lyapunov functions, Linear Matrix Inequalities (LMIs).

Consider the single-input single-output case for simplicity. Then applying the shift operators $z_{1}$ and $z_{2}$ to (as one choice among the various Fornasini-Marchesini models) in $(5)$ ( (under the assumption of zero boundary conditions) gives the 2D transferfunction representation

$$
Y\left(z_{1}, z_{2}\right)=G\left(z_{1}, z_{2}\right) U\left(z_{1}, z_{2}\right),
$$

where the transfer-function $G\left(z_{1}, z_{2}\right)$ is (as in the $1 \mathrm{D}$ systems case) the ration of two polynomials in $z_{1}$ and $z_{2}$ of the form

$$
G\left(z_{1}, z_{2}\right)=\frac{p\left(z_{1}, z_{2}\right)}{q\left(z_{1}, z_{2}\right)} .
$$

This representation has also been extensively used to study the stability of 2D linear systems, see, e.g., Huang (1972); Justice and Shanks (1973), starting from the 
necessary and sufficient condition

$$
q\left(z_{1}, z_{2}\right) \neq 0 \text { in } \mathcal{D},
$$

where in Huang (1972) it was shown that the requirement to check for all $\left(z_{1}, z_{2}\right)$ in $\mathcal{D}$ is equivalent to

1) $q\left(z_{1}, 0\right) \neq 0$, for all $\left|z_{1}\right| \leq 1$ and

ii) $q\left(z_{1}, z_{2}\right) \neq 0$, for all $\left|z_{1}\right|=1,\left|z_{2}\right| \leq 1$.

These conditions are also interchangeable in terms of $z_{1}$ and $z_{2}$.

Conditions i) and ii) allow the stability of a $2 \mathrm{D}$ discrete linear system to be determined by 1D linear systems stability tests, including those in the frequency domain. Condition i) should be tested first for a given example as it is a 1D stability test and a necessary condition. Condition ii) requires that the frequency response generated by $q\left(e^{j \omega_{1}}\right), \omega_{1} \in[0,2 \pi]$, lies inside the unit circle in the complex $z_{2}$ plane, which releases 1D linear systems frequency response methods for use in stability testing. By analogy with the 1D systems case, this should also extend to control law design.

Condition ii) requires frequency attenuation over the complete frequency spectrum. By analogy with the standard linear systems case, this may be overly stringent or not required since the performance requirements are only imposed over finite frequency ranges. This problem is discussed later in this paper.

A Lyapunov approach to stability analysis and control law design for 2D linear systems and repetitive processes has been the subject of extensive research and not restricted to single-input single-output examples. Two forms of Lyapunov equation for such systems are possible and these are termed, respectively, 1D and 2D. The first class uses a Lyapunov equation where the entries of the defining matrices are frequency dependent and gives necessary and sufficient conditions. By the 1D Lyapunov equation, the Fornasini-Marchesini state-space model (5) is stable if and only if there exists a matrix $P\left(e^{j \omega)}\right) \succ 0$ such that

$$
P\left(e^{j \omega} G\left(e^{j \omega}\right)+G^{T}\left(e^{-j \omega}\right) P\left(e^{j \omega}\right) \prec 0,\right.
$$

where

$$
G\left(z_{1}\right)=\left(I-z_{1} A_{1}\right)^{-1} A_{2}, \omega \in[0,2 \pi] .
$$

The 1D Lyapunov equation can be further developed to produce stability tests involving matrices with constant entries. This involves the matrix Krnoecher product and leads Agathoklis, Jury, and Mansour (1993) (where the analysis is for the Roesser model) to the requirement that a second order polynomial matrix of the form

$$
H(\lambda)=\lambda^{2} X_{1}+\lambda X_{2}+X_{3},
$$

where $\lambda=e^{j \omega}$ satisfies

$$
\operatorname{det} H(\lambda) \neq 0,
$$

for all $\omega \in[0,2 \pi]$. In the case of differential and discrete linear repetitive processes, the 1D equation also leads to bounds on performance Owens and Rogers (1995); Rogers 
and Owens (1993).

The alternative 2D Lyapunov equation is defined by matrices with constant entries and has an identical structure to the 1D linear systems case. For systems described by (5) the stability property holds provided these exists a matrix $P \succ 0$ such that Hinamoto (1993)

$$
Q=\left[\begin{array}{cc}
\beta P & 0 \\
0 & \gamma P
\end{array}\right]-A^{T} P A \prec 0
$$

where $\beta, \gamma>0, \beta+\gamma=1$ and $A=\left[\begin{array}{ll}A_{1} & A_{2}\end{array}\right]$.

A Lyapunov equation defined by matrices with constant entries has obvious attractions in terms of stability tests and control law design and was initially believed to be necessary and sufficient for stability but in Anderson, Agathoklis, Jury, and Mansour (1986) a counter-example and related analysis established that it was a sufficient but not necessary condition. In a number of special cases, however, it is necessary and sufficient, where the most physically relevant of these is single-input single-output examples. Also the 2D Lyapunov equation provides a starting point for the development of Linear Matrix Inequality (LMI) based stability tests and hence control law design algorithms and this is detailed below, after necessary and sufficient LMI stability tests are considered.

In Bliman (2002) LMI based necessary and sufficient conditions were developed for 2D linear systems described by the state-space model

$$
\begin{aligned}
& x_{1}(i+1, j)=A x_{1}(i, j)+B x_{2}(i, j), \\
& x_{2}(i, j+1)=C x_{1}(i, j)+D x_{2}(i, j),
\end{aligned}
$$

which is of the form (1) with the input and output terms deleted as internal stability is considered. Moreover, these results transfer to the Fornasini-Marchesini model and they are based on a class of quadratic Lyapunov functions, from which the 2D Lyapunov equation naturally arises. This link is detailed further next based on the notation used in Bliman (2002).

Introduce the quadratic Lyapunov function for (41) as

$$
V(i, j)=x_{1}^{T}(i, j) Q_{1} x_{1}(i, j)+V_{2}^{T}(i, j) Q_{2} x_{2}(i, j),
$$

where $Q_{1} \succ 0$ and $Q_{2} \succ 0$ are compatibly dimensioned matrices. Also introduce

$$
V_{11}(i, j)=x_{1}^{T}(i+1, j) Q_{1} x(i+1, j)+x_{2}^{T}(i, j+1) Q_{2} x_{2}(i, j+1)
$$

and hence

$$
V_{11}(i, j)-V(i, j)=\left[\begin{array}{l}
x_{1}(i, j) \\
x_{2}(i, j)
\end{array}\right]^{T} R\left[\begin{array}{l}
x_{1}(i, j) \\
x_{2}(i, j)
\end{array}\right]
$$

where

$$
R=\left[\begin{array}{cc}
A^{T} Q_{1} A-Q_{1} & A^{T} Q_{1} B \\
B^{T} Q_{1} A & B^{T} Q_{1} B
\end{array}\right]+\left[\begin{array}{cc}
C^{T} Q_{2} C & C^{T} Q_{2} D \\
D^{T} Q_{2} C & D^{T} Q_{2} D-Q_{2}
\end{array}\right]
$$

which gives the 2D Lyapunov equation for this case when $R \prec 0$. 
Remark 1. In some applications, the Lyapunov function (42) has physical meaning. This is the case for linear repetitive processes where the first term can be viewed as the 'energy' in the current pass state vector and the second the 'energy' in the previous pass profile.

To obtain necessary and sufficient conditions for stability, Bliman (2002) used a nonminimal state information where, instead of $x(i, j)$ as above, previous state vectors are used, i.e., $\left\{x^{T}(i, j) \quad x^{T}(i, j-1) \quad \ldots \quad x^{T}(i, j-h+1)\right\}$ where $h>0$ is an integer.

Introducing

$$
\begin{aligned}
\mathcal{X}_{1, h}(i, j) & =\left[\begin{array}{llll}
x_{1}^{T}(i, j) & x_{1}^{T}(i, j-1) & \vdots & x_{1}^{T}(i, j-h+1)
\end{array}\right]^{T}, \\
\mathcal{X}_{2, h}(i, j) & =\left[\begin{array}{llll}
x_{2}^{T}(i, j) & x_{2}^{T}(i, j-1) & \vdots & x_{2}^{T}(i, j-h+1)
\end{array}\right]^{T}
\end{aligned}
$$

and hence from (41)

$$
\begin{aligned}
& \mathcal{X}_{1, h}(i+1, j)=\left(I_{h} \otimes A\right) \mathcal{X}_{1, h}(i, j)+\left(I_{h} \otimes B\right) \mathcal{X}_{2, h}(i, j,) \\
& \mathcal{X}_{2, h}(i, j+1)=\left(I_{h} \otimes C\right) \mathcal{X}_{1, h}(i, j)+\left(I_{h} \otimes D\right) \mathcal{X}_{2, h}(i, j .) .
\end{aligned}
$$

Also introduce

$$
V_{h}(i, j)=\mathcal{X}_{1, h}^{T}(i, j) Q_{1, h} \mathcal{X}_{1, h}(i, j)+\mathcal{X}_{2, h}^{T} \mathcal{X}_{2, h}(i, j),
$$

for some $Q_{1, h} \succ 0$ and $Q_{2, h} \succ 0$. Then (44) holds with obvious substitutions for the variables where

$$
\begin{aligned}
R & =\left[\begin{array}{cc}
\left(I_{h} \otimes A\right)^{T} Q_{1, h}\left(I_{h} \otimes A\right)-Q_{1, h} & \left(I_{h} \otimes A\right)^{T} Q_{1, h}\left(I_{h} \otimes B\right) \\
\left(I_{h} \otimes B\right)^{T} Q_{1, h}\left(I_{h} \otimes A\right) & \left(I_{h} \otimes B\right)^{T} Q_{1, h}\left(I_{h} \otimes B\right)
\end{array}\right] \\
+ & {\left[\begin{array}{cc}
\left(I_{h} \otimes C\right)^{T} Q_{2, h}\left(I_{h} \otimes C\right) & \left(I_{h} \otimes C\right)^{T} Q_{2, h}\left(I_{h} \otimes D\right) \\
\left(I_{h} \otimes D\right)^{T} Q_{1, h}\left(I_{h} \otimes C\right) & \left(I_{h} \otimes D\right)^{T} Q_{2, h}\left(I_{h} \otimes D\right)-Q_{2, h}
\end{array}\right] . }
\end{aligned}
$$

Only trajectories of (41) that satisfy (47) are of interest and hence there are constraints on $\mathcal{X}_{1, h}$ and $\mathcal{X}_{2, h}$ as exploited in Bliman (2002) to form a particular version of the matrix $R$ in the 2D Lyapunov equation that increases in dimension with $h$ and let this matrix be denoted by $R_{h}$. Then the following is the central result in Bliman (2002).

Theorem 4.3. The following are equivalent.

(1) A $2 D$ system described by (41) is asymptotically stable.

(2) For any $\left(z_{1}, z_{2}\right) \in \mathbb{C}^{2},\left|z_{1}\right| \leq 1,\left|z_{2}\right| \leq 1 \Rightarrow$

$$
\operatorname{det}\left(\left[\begin{array}{cc}
I-z_{1} A & -z_{1} B \\
-z_{2} C & I-z_{2} D
\end{array}\right]\right) \neq 0
$$

(3) $\rho(D)<1$ and for any $z \in \mathbb{C}$

$$
|z| \leq 1 \Rightarrow \rho\left(A-z(I-z D)^{-1} C\right)<1
$$


(4) $\rho(A)<1$ and for any $z \in \mathbb{C}$

$$
|z| \leq 1 \Rightarrow \rho\left(z C(I-z A)^{-1} B+D\right)<1 .
$$

(5) There exists $h^{*}$ such that $R_{h}^{*} \prec 0$.

(6) There exists $h^{*}$ such that $R_{h}^{*} \prec 0$ for all $h \geq h^{*}$.

This result gives a family of LMI conditions for asymptotic stability. In particular, a 2D system is asymptotically stable if and only if there exists a Lyapunov function of form (48), generalizing (42) and decreasing along the system trajectories. Moreover, the conditions in this last result are easy to check but the problem is that there is no a priori formula for computing an upper bound on $h$.

This result is of a similar form to that given in Fornasini and Marchesini (1980) for (2) with no inputs, resulting in the following necessary and sufficient condition for stability

$$
\sum_{i+j=h}\left\|A_{1}{ }^{i} \uplus^{j} A_{2}\right\|<1
$$

where $A_{1}{ }^{i} \omega^{j} A_{2}$ denotes the suffle product, i.e., $i$ times the matrix $A_{1}$ and $j$ times the matrix $A_{2}$. The difficulty with this result is that there is no method to determine a priori the number of operations required to check the stability of a given example. This result does, however, provide a family of sufficient conditions for stability whose conservativeness vanishes asymptotically.

Another set of necessary and sufficient stability conditions follows by using the following result from Fornasini and Marchesini (1980).

Lemma 4.4. The following are equivalent conditions

i) A $2 D$ system described by (2) is asymptotically stable.

ii)

$$
\operatorname{det}\left(I-z_{1} A_{1}-z_{2} A_{2}\right) \neq 0, \text { for all }\left|z_{1}\right| \leq 1,\left|z_{2}\right| \leq 1 \text {. }
$$

iii)

$$
\rho(A(\theta))<1, \text { for all } \theta \in[0,2 \pi]
$$

where

$$
A(\theta)=A_{1}+e^{j \theta} A_{2} .
$$

The LMI based tests of Ebihara, Ito, and Hagiwara (2006) require the concept of a Guardian map, see, e.g., Barmish (1994), denoted by $\nu$, for the set of Schur stable, i.e., all eigenvalues have modulus strictly less than unity, complex matrices. In the case of $q \times q$ matrix, say $M$,

$$
\nu(M)=\operatorname{det}\left(\bar{M} \otimes M-I_{n} \otimes I_{n}\right),
$$


where $\bar{M}$ is formed by taking the complex conjugate of each entry in $M$.

Using this map, the following is Lemma 2 in Ebihara et al. (2006)

Lemma 4.5. A $2 D$ system described by (2) is asymptotically stable if and only if i)

$$
\rho\left(A_{1}+A_{2}\right)<1,
$$

ii)

$$
\operatorname{det} \mathcal{A}(\theta) \neq 0, \text { for all } \theta \in[0,2 \pi]
$$

where

$$
\begin{aligned}
\mathcal{A}(\theta) & =\bar{A}(\theta) \otimes A(\theta)-I \otimes I \\
& =-\mathcal{A}_{-1} e^{-j \theta}+\mathcal{A}_{0}+\mathcal{A}_{1} e^{j \theta}, \\
\mathcal{A}_{0} & =\bar{A}_{1} \otimes A_{1}+\bar{A}_{2}-I \otimes I, \\
\mathcal{A}_{-1} & =\bar{A}_{2} \otimes A_{1}, \\
\mathcal{A}_{1} & =\bar{A}_{1} \otimes A_{2} .
\end{aligned}
$$

The next stage exploits the link between parameter dependent LMIs to parameterindependent equivalents using the discrete-time positive real lemma.

Introduce the following for this last set of equations

$$
\begin{aligned}
\mathcal{G}_{0} & =\frac{1}{2}\left(\mathcal{A}_{-1}^{*} \mathcal{A}_{-1}+\mathcal{A}_{0}^{*} \mathcal{A}_{0}+\mathcal{A}_{1}^{*} \mathcal{A}_{1}\right), \\
\mathcal{G}_{-1} & =\mathcal{A}_{0}^{*} \mathcal{A}_{-1}+\mathcal{A}_{1}^{*} \mathcal{A}_{0} \\
\mathcal{G}_{-2} & =\mathcal{A}_{1}^{*} \mathcal{A}_{-1}
\end{aligned}
$$

Then the following is Theorem 1 in Ebihara et al. (2006).

Theorem 4.6. A $2 D$ system described by (2) is asymptotically stable if and only if

i) (54) holds,

ii) There exist matrices $Q_{11}, Q_{22}$ and $Q_{12}$ such that

$$
\left[\begin{array}{ccc}
0 & 0 & \mathcal{G}_{-2}^{*} \\
0 & 0 & \mathcal{G}_{-1}^{*} \\
\mathcal{G}_{-2} & \mathcal{G}_{-1} & \mathcal{G}_{0}+\mathcal{G}_{0}^{*}
\end{array}\right] \succ\left[\begin{array}{ccc}
Q_{11} & Q_{12} & 0 \\
Q_{12}^{*} & Q_{22}-Q_{11} & -Q_{12} \\
0 & -Q_{12}^{*} & -Q_{22}
\end{array}\right]
$$

This last condition is necessary and sufficient, as opposed to sufficient, and hence stability tests based on it should be less conservative than the alternative above. A remaining problem is the dimension of the LMI in (58) which is $3 n^{2}$ and the number of complex scalar variables is $n^{2}\left(2 n^{2}+1\right)$. Hence it is not possible to apply this test for a large dimensioned example. To reduce the computational cost, LMIs of smaller dimensions results can be derived using the generalized $\mathcal{S}$ procedure, see, .e.g, Ebihara et al. (2006), leading to the following result established as Theorem 2 in Ebihara et al. (2006).

Theorem 4.7. A 2D system described by (2) is asymptotically stable if and only if 
i) (54) holds,

ii) there exists matrices $Q_{11}, Q_{22}$ and $Q_{12}$ such that

$$
\left[\begin{array}{c}
\mathcal{A}_{-1}^{T} \\
\mathcal{A}_{0}^{T} \\
\mathcal{A}_{1}^{T}
\end{array}\right]^{\perp}\left[\begin{array}{ccc}
Q_{11} & Q_{12} & 0 \\
Q_{12} & Q_{22}-Q_{11} & -Q_{12} \\
0 & -Q_{12} & -Q_{22}
\end{array}\right]\left[\begin{array}{c}
\mathcal{A}_{-1}^{T} \\
\mathcal{A}_{0}^{T} \\
\mathcal{A}_{1}^{T}
\end{array}\right]^{\perp^{*}} \prec 0,
$$

where $\perp$ denotes the orthogonal complement.

This result has the same number of scalar variables as (58) but the dimension of the LMI has been reduced from $3 n^{2}$ to $3 n^{2}-r$, where $r=\operatorname{rank}\left[\begin{array}{ccc}\mathcal{A}_{1} & \mathcal{A}_{0} & \mathcal{A}_{1}\end{array}\right]^{T}$. Moreover, if $\mathcal{A}_{0}$ or $\mathcal{A}_{1}$ is nonsingular, the LMI dimension is $2 n^{2}$ and furtheer development is possible.

The condition of (51) is equivalent to

$$
\operatorname{det}\left(I-\left[\begin{array}{cc}
z_{1} I & 0 \\
0 & z_{2} I
\end{array}\right]\left[\begin{array}{l}
I \\
I
\end{array}\right]\left[\begin{array}{ll}
A_{1} & A_{2}
\end{array}\right] \neq 0 \text {, for all }\left|z_{1}\right| \leq 1,\left|z_{2}\right| \leq 1 .\right.
$$

and applying $D$-scaling with scaling matrix

$$
W=\left[\begin{array}{cc}
W_{1}^{\frac{1}{2}} & 0 \\
0 & W_{2}^{\frac{1}{2}}
\end{array}\right], W_{1} \succ 0, W_{2} \succ 0,
$$

gives that asymptotic stability holds if

$$
\left[\begin{array}{cc}
A_{1}^{T}\left(W_{1}+W_{2}\right) A_{1}-W_{1} & A_{1}^{T}\left(W_{1}+W_{2}\right) A_{2} \\
A_{2}^{T}\left(W_{1}+W_{2}\right) A_{1} & A_{2}^{T}\left(W_{1}+W_{2}\right) A_{2}-W_{2}
\end{array}\right] \prec 0, W_{1} \succ 0, W_{2} \succ 0 .
$$

This LMI condition was developed in, e.g., Galkowski, Rogers, Xu, Lam, and Owens (2002); Ooba (2000) without $D$-scaling and has proved the basis for iterative learning control law designs that have been experimentally verified, e.g., Hladowski et al. (2010, 2012), Paszke, Rogers, and Galkowski (2016), where the latter result also makes use of the generalized KYP lemma. This topic and, in particular, the debate about use of sufficient as opposed to necessary and sufficient stability conditions, is returned to in a later section of this paper on control law design, where more recent work Bachelier, Paszke, Yeganefar, Mehdi, and Cherifi (2016, 2017); Chesi and Middleton (2014) on necessary and sufficient stability conditions will also be discussed.

\section{Systems Theoretic Properties - State-space and System Matrix Approaches}

As in the case of 1D linear systems, an extensive volume of research has considered the definition and characterization of properties such as controllability, observability, system equivalences poles and zeros and realization theory. The analysis has used the state-space and polynomial settings for analysis. One desirable feature of such theory is that it should link to control/regulation theory. For example, is it the case for 2D linear systems that controllability gives a solution to pole placement (if such a concept 
exists) as in the 1D linear systems case? This section gives an overview of the main results currently available with particular emphasis on the link to control/regulation of the dynamics.

\subsection{State-space Model Analysis}

Controllability for 2D linear systems can be split into local, which refers to a single local state, and global, which refers to infinite sets of local states lying on a separation set, where for 1D systems, the separation between past and future is given by a single time instant, i.e., a point. Hence the recursive computation of a 1D system trajectory consists of updating values on successive points of the domain. The situation is more complex in the $2 \mathrm{D}$ case, see also the discussion re separation sets associated with Figure 2.

One way to define these properties is in terms of the state dynamics and the following partial ordering of two tuple integers is required.

$$
\begin{aligned}
& (i, j) \leq\left(p_{1}, p_{2}\right) \quad \text { iff } i \leq p_{1}, j \leq p_{2} \\
& (i, j)=\left(p_{1}, p_{2}\right) \quad \text { iff } i=p_{1}, j=p_{2}, \\
& (i, j)<\left(p_{1}, p_{2}\right) \quad \text { iff } \quad(i, j) \leq\left(p_{1}, p_{2}\right), \quad(i, j) \neq\left(p_{1}, p_{2}\right) .
\end{aligned}
$$

Any of the Fornasini-Marchesini models is said to be locally reachable in the rectangle $\left[(0,0),\left(p_{1}, p_{2}\right)\right]$ if, corresponding to any initial states and any state $z^{*} \in \mathbb{R}^{n}$, there exist control input sequence $u(i, j)$ on $(0,0) \leq(i, j) \leq\left(p_{1}, p_{2}\right)$ such that $z\left(p_{1}, p_{2}\right)=z^{*}$. Choosing $z^{*}$ as the zero vector defines the controllability property. Moreover, one method of obtaining conditions for the existence of this property is to use the 2D systems version of the transition matrix, also termed the fundamental matrix sequence in some of the literature, see, e.g., Kaczorek (1985).

A great many results have been produced based on this approach and in this paper one example is given. Consider the Fornasini Marchesini model (25), which also has been be used in the analysis of discrete linear repetitive processes. The fundamental matrix sequence, denoted by $T_{n_{1}, n_{2}}$, is Gałkowski, Rogers, and Owens (1998)

$$
E T_{i, j}=\left\{\begin{array}{cc}
\widetilde{A}_{3} T_{-1,-1}+\widetilde{A}_{1} T_{0,-1}+I & i=j=0 \\
\widetilde{A}_{3} T_{i-1, j-1}+\widetilde{A}_{1} T_{i, j-1} & i \neq 0 \text { and } / \text { or } j \neq 0
\end{array} .\right.
$$

and the following result gives conditions for local controllability and reachability Gałkowski et al. (1998).

Theorem 5.1. The singular $2 D$ Fornasini Marchesini state-space model (25) is locally reachable in the rectangle $\left[(0,0),\left(p_{1}, p_{2}\right)\right]$ if and only if

$$
\operatorname{rank} R_{p_{1}, p_{2}}=n,
$$

where

$$
R_{p_{1}, p_{2}}=\left[\begin{array}{lllllll}
M_{0,-1} & M_{0,0} & \cdots & M_{0, p_{2}-1} & M_{1,-1} & \cdots & M_{p_{1}-1, p_{2}-1}
\end{array}\right]
$$


and

$$
M_{i, j}=T_{i, j} \widetilde{B}_{1}, \quad 1 \leq i \leq p_{1}, \quad 1 \leq j \leq p_{2} .
$$

Unlike the 1D systems case, analysis of this form has found very limited use in formulation and solution of control problems.

Another form of controllability and observability is termed global. Consider the time set $T=\mathbb{Z} \times \mathbb{Z}$, where $\mathbb{Z} \times \mathbb{Z}$ has the partial order stated above, and the past of the point $\left(n_{1}, n_{2}\right)$ is $\mathcal{P}\left(n_{1}, n_{2}\right)=\left\{(l, m):(l, m) \leq\left(n_{1}, n_{2}\right)\right\}$ and the future is $\mathcal{F}(i, j)=$ $\{(l, m):(i, j) \leq(l, m)\}$. Let $\mathcal{U}$ be the space of past inputs for the example considered, $\mathcal{Y}$ the space of future outputs, and $\mathcal{X}$ the global state. Then the controllability map, denoted by $\mathcal{C}$, is

$$
\mathcal{C}: u(\cdot, \cdot) \in \mathcal{U} \rightarrow \mathcal{X}_{(0,0)} \in \mathcal{X},
$$

and the observability map is

$$
\Theta: \mathcal{X}_{(0,0)} \in \mathcal{X} \rightarrow \mathcal{Y}_{(\cdot, \cdot)} \in \mathcal{Y}
$$

The example under consideration is globally controllable if and only if $\mathcal{C}$ is a surjective map, and globally observable if and only if $\Theta$ is an injective map. In the 1D case, these global concepts are closely linked to the properties of surjectivity and injectivity for the state-space factorization of the input-output map Kalman, Falb, and Arbib (1969).

For 2D systems, again there is no link with minimality of the state-space model or to a direct link with the existence of control laws. In particular, there is no counterpart of the $1 \mathrm{D}$ systems result that controllability of the state-space model is a necessary and sufficient condition for the existence of a stabilizing state feedback control law. In Gałkowski et al. (1998) the singular Fornasini-Marchesini state-space model representation is used to obtain necessary and sufficient conditions for the properties of local reachability and controllability of discrete linear repetitive processes in terms of matrix rank based tests. For discrete linear repetitive processes there is also the property of pass profile controllability that has no Roesser or Fornasini Marchesini state-space model equivalent but does 'recover' the 1D systems result that one form of stabilization for these properties is possible if and only if pass profile controllability holds Dymkov, Gaishun, Rogers, Galkowski, and Owens (2003).

\subsection{Systems Matrix Approaches}

The concept of strict system equivalence is fundamental to the study of $1 \mathrm{D}$ linear dynamics. The original work for such systems is given in (Rosenbrock, 1970) with a subsequent generalization in Fuhrmann (1977). This property describes the connection between all least order realizations of a transfer-function matrix, starting the polynomial system

$$
\begin{aligned}
T x & =U u, \\
y & =V x+W u,
\end{aligned}
$$

where $T, U, V$ and $W$ are polynomial matrices in one indeterminate, arising from a set of linear differential or difference equations. 
The strict system equivalence property has also been studied for 2D systems, see, e.g., Frost and Boudellioua (1989) and Pugh, McInerney, Boudellioua, Johnston, and Hayton (1998), where the underlying approach was to generalize the results for the 1D systems case, for which the background can be found in, e.g., Rosenbrock (1970). This approach starts from the natural generalization of (70), i.e.,

$$
\begin{aligned}
T\left(z_{1}, z_{2}\right) x & =U\left(z_{1}, z_{2}\right) u \\
y & =V\left(z_{1}, z_{2}\right) x+W\left(z_{1}, z_{2}\right) u
\end{aligned}
$$

where $x \in \mathbb{R}^{n}$ is the state vector, $u \in \mathbb{R}^{p}$ is the input vector and $y \in \mathbb{R}^{m}$ is the output vector, $T, U, V$ and $W$ are polynomial matrices with elements in $\mathbb{R}\left[z_{1}, z_{2}\right]$ of dimensions $r \times r, r \times p, m \times l$ and $m \times p$ respectively.

Using (71), the system matrix in the general form is:

$$
P\left(z_{1}, z_{2}\right)=\left[\begin{array}{rr}
T\left(z_{1}, z_{2}\right) & U\left(z_{1}, z_{2}\right) \\
-V\left(z_{1}, z_{2}\right) & W\left(z_{1}, z_{2}\right)
\end{array}\right]
$$

where

$$
P\left(z_{1}, z_{2}\right)\left[\begin{array}{r}
x \\
-u
\end{array}\right]=\left[\begin{array}{r}
0 \\
-y
\end{array}\right]
$$

If $T\left(z_{1}, z_{2}\right)$ is invertible, the system matrix in (72) is said to be regular. In this case the transfer-function matrix corresponding to the system matrix in (72) is given by

$$
G\left(z_{1}, z_{2}\right)=V\left(z_{1}, z_{2}\right) T^{-1}\left(z_{1}, z_{2}\right) U\left(z_{1}, z_{2}\right)+W\left(z_{1}, z_{2}\right) .
$$

In the particular case of the Fornasini-Marchesini state-space model (2), with $D=0$ for simplicity,

$$
P\left(z_{1}, z_{2}\right)=\left[\begin{array}{cc}
z_{1} z_{2} I-z_{1} A_{1}-z_{2} A_{2} & z_{1} B_{2}+z_{2} B_{2} \\
-C & 0
\end{array}\right] .
$$

Generalizing 1D systems results directly to $n \mathrm{D}$ systems algebraically is, in general, non-trivial due, see also the discussion in the introduction of this paper, to significant differences between the ring of polynomials in one indeterminate and that in two or more. Also $n \mathrm{D}$ systems results developed for one model may not transfer to another and results that hold for $2 \mathrm{D}$ systems may not hold for $n \mathrm{D}$ systems, where $n>2$.

In the transfer function description of the dynamics a 1D differential or discrete linear system a common factor between the numerator and denominator polynomials is a pole-zero cancelation and its effects on subsequent analysis are understood. The absence of common factors for two or more polynomials means that they are coprime. Also equivalent characterizations of the coprimeness property exist, including the Bezout identity.

Algebraically, 1D polynomial-based systems theory is underpinned by ring-theory, where polynomials in one indeterminate have a division algorithm for Euclidean rings. This result forms the basis for the algorithmic derivation of canonical forms and solution techniques in 1D systems theory, such as the Smith form, and the solution of 1D polynomial equations, see, e.g., Kailath (1980). In $n \mathrm{D}$ linear systems, see the introduction section of this paper, three forms of coprimeness, namely, factor, minor, and 
zero, respectively, exist and two are distinct properties for $n=2$ and for $n>3$ all are distinct properties.

Another standard tool in 1D systems theory is that elementary matrices are a sub-class of unimodular matrices over a ring, where all unimodular matrices can be formed as a product of elementary matrices. For rings, $F\left[z_{1}, z_{2}, \cdots, z_{n}\right], n \geq 2$, not all unimodular matrices can be formed as a product of elementary matrices. In the case $n \geq 2$ progress is possible by treating each polynomial ring as a sub-ring of a larger ring with a division algorithm. The mechanism is to favor one of the indeterminates and consider elements of the ring to be polynomial in this indeterminate with coefficients rational in the other indeterminates.

Despite the analysis problems summarized above, progress has been reported in a number of areas, where, as examples, the following are considered.

Definition 5.2. (Pugh et al., 1998) Let $\mathbb{T}(m, n)$ denote the class of $(r+m) \times(r+n)$ rational matrices where $r>\min (m, n)$. The subset $\mathbb{P}(m, n)$ of $\mathbb{T}(m, n)$ obtained by requiring $r>0$ represents the $2 \mathrm{D}$ rational system matrices. Two system matrices $P_{1}\left(z_{1}, z_{2}\right)$ and $P_{2}\left(z_{1}, z_{2}\right) \in \mathbb{P}(m, n)$, are said to be $\mathrm{I} / \mathrm{O}$ equivalent if they have the same transfer-function matrix, i.e.,

$$
G_{1}\left(z_{1}, z_{2}\right)=G_{2}\left(z_{1}, z_{2}\right) .
$$

Definition 5.3. Two admissible systems $\left(T_{i}, U_{i}, V_{i}, W_{i}\right)$ are said to be system equivalent if there exist rational matrices $Q_{l}, R_{l}, Q_{r}, R_{r}$ of compatible dimensions such that

$$
\left[\begin{array}{ll}
Q_{l} & 0 \\
R_{l} & I
\end{array}\right]\left[\begin{array}{cc}
T_{1} & U_{1} \\
-V_{1} & W_{1}
\end{array}\right]=\left[\begin{array}{cc}
T_{2} & U_{2} \\
-V_{2} & W_{2}
\end{array}\right]\left[\begin{array}{cc}
Q_{r} & R_{r} \\
0 & I
\end{array}\right]
$$

The following result, Theorem 1 in Zerz (2000a), generalizes the 1D result that systems equivalence is the same as equality of the transfer-functions.

Theorem 5.4. Two admissible systems are realizations of the same transfer-function is and only if they are system equivalent.

Strict system equivalence is defined as follows, Definition 4 in (Zerz, 2000a).

Definition 5.5. Two admissible systems $\Sigma_{1}$ and $\Sigma_{2}$ are strictly system equivalent in the sense of Rosenbrock if (77) holds with polynomial interwinning matrices and $Q_{l}$ and $Q_{r}$ that are unimodular.

Theorem 5.6. Two systems that are FSSE give rise to the same transfer-function and have the same determinantal ideal.

In the case of 2D systems with $n>2$ further complications arise and for this case Zerz (2000b) gives a comprehensive treatment and is also a starting point for results obtained after the publication of this monograph. 


\section{Systems Theoretic Properties - Behavioral Analysis}

\subsection{Background}

As described in Section 2.4, in the behavioral systems setting for the representation and analysis of linear systems the central object of study is the behavior $\mathcal{B}$, i.e., the set of trajectories that satisfy the laws of the system, the properties of $\mathcal{B}$, and how such properties are reflected in properties of the representations of $\mathcal{B}$. Linear difference behaviors are defined as the solution set of a system of linear partial difference equations. Equivalently, a linear difference behavior $\mathcal{B}$ can be interpreted as the kernel of a polynomial partial difference operator represented by a polynomial matrix. In the $2 D$ case $\mathcal{B}=\operatorname{ker} R\left(z_{1}, z_{2}, \hat{z}_{2}\right)$, where $R$ is a $p \times q$ Laurent polynomial matrix in the indeterminates $z_{1}, z_{2}, \hat{z}_{1}, \hat{z}_{2}$. This setting for the discrete case also extends to continuous-time systems.

The representation (70), which includes the Fornasini Marchesini model as a particular case, can be treated as a behavior in the kernel form as

$$
\mathcal{B}_{x, u, y}=\operatorname{ker}_{\mathcal{A}}\left(\begin{array}{ccc}
T & -U & 0 \\
V & W & -I
\end{array}\right),
$$

where $\mathcal{A}$ is the signal space and is a vector space over a field, which is taken to be $\mathbb{R}$ or $\mathbb{C}$. Use of this setting has led to a very large volume of results on properties such as controllability, observability, poles and zeros and control by interconnection.

The behavioral approach to systems analysis draws on very powerful algebraic tools in module theory and especially the theory of finitely generated modules, i.e., a finite generating set, modules over a ring, where for further background if required, see, e.g., Lang (1997). Moreover, a duality theory exists see, e.g., Malgrange (1962/63); Oberst (1990). Next some consequences of this theory relative to $2 \mathrm{D}$ (and $n \mathrm{D}$ ) systems theory are given (with others related to stabilization considered in Section 8).

\subsection{Controllability, Obervability and Input-Output Representations}

Consider a 1D linear system with kernel representation $\mathcal{B}=\operatorname{ker} R$. Then controllability Polderman and Willems (1998) is defined as an intrinsic system property that does not depend on the choice of a particular representation. The behavior $\mathcal{B}$ is termed controllable if for all $w_{1}, w_{2} \in \mathcal{B}$ there exists a $T$ and $w \in \mathcal{B}$ such that

$$
w(t)=\left\{\begin{array}{l}
w_{1}(t), \quad t \leq 0 \\
w_{2}(t), \quad t \geq t_{1}
\end{array},\right.
$$

for which equivalent definitions exist. This property implies that within the behavior it is possible to switch form one trajectory to another provided a delay is allowed.

The 2D systems definition of controllability in the behavioral setting, see, e.g. Wood, Rogers, and Owens (1999) is the natural generalization of the 1D case. Moreover, a comprehensive set of results is available on conditions for its existence. Likewise for observability, which for a 1D linear system in the behavioral (Polderman \& Willems, 1998) setting starts from a partition (where $z$ denotes the forward shift operator in 1D linear systems theory)

$$
R_{1}(z) w_{1}=R_{2}(z) w_{2}
$$


of the system laws $R(z) w=0$, and $w_{2}$ is said to be observable from $w_{1}$ if knowledge of $w_{1}$ yields knowledge of $w_{2}$. Equivalently, by linearity, $w_{1}=0$ implies $w_{2}=0$ or $R_{2}$ is right-prime. The definition of observability from (80) generalizes, see. e.g., Rocha (1990) to $2 \mathrm{D}$ behaviors.

In a partition $w=\left[\begin{array}{ll}w_{1}^{T} & w_{2}^{T}\end{array}\right]^{T}$ of the system variables with corresponding decomposition $R_{1} w_{1}=R_{2} w_{2}$ of the system laws, $w_{2}$ is termed strongly observable from $w_{1}$ if it is uniquely determined by $w_{1}$. Equivalently, $R_{2}$ is zero right-prime. A weaker observability definition arises when $R_{2}$ is a right factor-prime matrix, where $w_{1}$ determines $w_{2}$ up to a finite-dimensional real vector space, see, e.g., Rocha and Willems (1991); Wood et al. (1999); Zerz (2000b).

Input-output structures in the behavioral setting have an algebraic characterization and if $(u, y)$ is an input-output structure then, given a polynomial matrix $R$ such that $\mathcal{B}=$ ker $R$, there exists Polderman and Willems (1998) a permutation $\Pi$ of the columns of $R$ such that $R \Pi=\left[\begin{array}{cc}-Q & P\end{array}\right]$, where $P$ is full column rank. The columns of $Q$ correspond to the input variables $u$, and the columns of $P$ to the output variables $y$. Moreover, the number of inputs is necessarily equal to $m(\mathcal{B})$, the maximum size of a set of free variables, which is independent of the input-output structure. From the definition of input and output and from the input-output representation, the definition of a transfer-function matrix $G$ is

$$
P G=Q
$$

Quarter-plane causality of the Fornasini-Marchesini state-space model described by (25) imposes the condition that

$$
y(i, j)=\sum_{l=-\infty}^{i} \sum_{k=-\infty}^{j} h(i-l, j-k) u(i, j),
$$

i.e., a particular structure or preferred direction of updating on the computation of the state and output vectors. In the case when all variables of an $2 \mathrm{D}$ (or $n \mathrm{D}, n \geq 3$ ) system are temporal, the causality requirement can be removed by allowing singular systems models.

Treating the concept of state in a behavioral setting for $2 \mathrm{D}$ (or $n \mathrm{D}, n \geq 3$ ) systems leads to first-order state-space models without a preferred direction, which, in turn, requires a formalization of the concepts of past, future, and of the independence of the future of a trajectory given the past, see, e.g. Rocha and Willems (1991); Zerz (2000b). In the 2D systems case the result is discrete state-space models defined by equations with the structure

$$
\begin{aligned}
E_{1} x+F_{1} z_{1} x & =0 \\
E_{2} x+G_{2} z_{2} x & =0 \\
E_{3} x+F_{3} z_{1} x+G_{3} z_{2} x+H_{3} z_{1} z_{2} x & =0 \\
N x+M w & =0
\end{aligned}
$$

where $x$ is the state variable vector and the matrices $E_{1}, E_{2}, E_{3}, F_{1}, F_{3}, G_{2}, G_{3}, H_{3}, M$, and $N$ have additional properties Rocha and Willems (1989). The state equations (83), (84), and (85) are first-order in $x$ and zeroth order in $w$, where these properties are not postulated a priori as is the case for both the Roesser and Fornasini-Marchesini 
state-space models. Instead they are a consequence of the Markovianity of the variable $x$.

The behavioral setting can also be extended to systems whose dynamics are not restricted to the positive quadrant of the $2 \mathrm{D}$ plane and consequently a stability theory is required for systems not restricted to this quadrant. Using behavioral representations of the dynamics, it is possible to consider systems that evolve over all four quadrants (if required). Moreover, one way to obtain a more generally applicable stability theory is to be more flexible in the prescription of past and future of such trajectories. It is possible to give a meaningful definition of stability for behaviors, where trajectory evolution is not restricted to the positive quadrant, using proper characteristic cones Valcher (2000) as a setting for analysis.

\subsection{Poles and Zeros}

Behavioral theory gives a definition Wood, Oberst, Rogers, and Owens (2000) of a finite pole of a linear system that agrees with the 1D linear systems case and allows a direct extension to $n \mathrm{D}$ linear systems, where a division of the poles into controllable and uncontrollable is possible. In the case of linear repetitive processes, the behavioral definition of a pole produces a generalization of the exponential frequency interpretation of a pole for 1D linear systems.

Consider the 2D polynomial matrix

$$
P\left(z_{1}, z_{2}\right)=\left[\begin{array}{cc}
T & -U \\
V & W
\end{array}\right],
$$

arising from a $2 \mathrm{D}$ linear system. Then a possible characteristic polynomial is

$$
\operatorname{det} P\left(z_{1}, z_{2}\right)=0 \text {, }
$$

and the reasoning is that (87) defines the poles of the 2D linear system.

Definition 6.1. The poles of a $2 \mathrm{D}$ discrete linear systems are the component-wise nonzero points in $2 \mathrm{D}$ complex space where the matrix of (87) fails to have full rank, i.e., they are given by the set

$$
\mathcal{V}=\left\{\left(a_{1}, a_{2}\right) \in \mathbb{C}^{2} \mid \operatorname{det} \rho\left(a_{1}, a_{2}\right)=0\right\} .
$$

The pole variety $\mathcal{V}$ for $2 \mathrm{D}$ linear system is given by the vanishing of a single $2 \mathrm{D}$ non-unit polynomial, and is therefore guaranteed to be a one-dimensional geometric set in 2D complex space, i.e., a curve. In particular, the pole variety cannot be zerodimensional, i.e., finite. The pole variety is a complex variety, even though the entries of the matrices defining the state-space model are real and is required to capture the full exponential-type dynamics of the system.

Assume that $\left(a_{1}, a_{2}\right) \in \mathbb{C}^{2}$ is such that (88) is satisfied for $z_{1}=a_{1}$ and $z_{2}=a_{2}$, and write $a_{1}=r_{1} e^{\imath \theta_{1}}, a_{2}=r_{2} e^{\imath \theta_{2}}$, with $r_{1}=0$ for $a_{1}=0$ and $r_{2}=0$ for $a_{2}=0$. Equivalently, discrete linear repetitive process under consideration has Wood et al. 
(2000) an exponential trajectory of the form

$$
\begin{aligned}
x_{k}^{\prime}(p) & =x_{00}^{1} r_{1}^{p} r_{2}^{k} \cos \left(\theta_{1} p+\theta_{2} k\right) \\
& +x_{00}^{2} r_{1}^{p} r_{2}^{k} \sin \left(\theta_{1} p+\theta_{2} k\right), \\
y_{k}^{\prime}(p) & =y_{00}^{1} r_{1}^{p} r_{2}^{k} \cos \left(\theta_{1} p+\theta_{2} k\right) \\
& +y_{00}^{2} r_{1}^{p} r_{2}^{k} \sin \left(\theta_{1} p+\theta_{2} k\right), \\
u_{k}^{\prime}(p) & =0,
\end{aligned}
$$

where $x_{00}^{1}, x_{00}^{2} \in \mathbb{R}^{n}, y_{00}^{1}, y_{00}^{2} \in \mathbb{R}^{m}$, and at least one of these four is nonzero. The form of exponential trajectory arising can be characterized algebraically Oberst (1995). Conversely, the existence of such a trajectory implies that $\rho\left(r_{1} e^{\imath \theta_{1}}, r_{2} e^{\imath \theta_{2}}\right)=0$, i.e., the frequency $\left(r_{1} e^{\imath \theta_{1}}, r_{2} e^{\imath \theta}\right)$ is a pole of the system.

Consider a discrete linear repetitive process described by (8). The using (90), (91), and (92)in this case, it follows that if $\left|a_{2}\right|=r \geq 1$ then a nonzero exponential, or sinusoidal, state-output trajectory exists in the system, which tends to infinity as the pass number increases but may remain stable along each given pass. Conversely, if $\left|a_{2}\right|=r<1$ for all poles $\left(a_{1}, a_{2}\right)$, then no trajectory tends to infinity for a given value of $p$ as the pass number increases, but there may be trajectories tending to infinity along-the-pass. In order to exclude trajectories of the form (90), (91), and (92) that are unstable either along-the-pass or in the $k$-direction, poles $\left(a_{1}, a_{2}\right)$ with $\left|a_{1}\right| \geq 1$ must also be excluded. Equivalently, the characteristic variety (89) of the zero-input behavior must lie inside

$$
\mathcal{P}_{1}:=\left\{\left(z_{1}, z_{2}\right) \in \mathbb{C}^{2}:\left|z_{1}\right| \leq 1,\left|z_{2}\right| \leq 1\right\}
$$

Hence stability is equivalent to all poles lying in $\mathcal{P}_{1}$.

The use of the behavioral setting to define zeros for $n \mathrm{D}$ linear systems and repetitive processes leads to a generalization of the blocking frequency interpretation from 1D linear systems, see, e.g. Zaris, Wood, and Rogers (2001).

\section{Other Systems Theoretic Analysis}

Realization theory is a central part of 1D linear systems theory and a basic result is that a state-space realization constructed from a transfer-function (or transfer-function matrix) is minimal if and only if it is controllable and observable. This problem is much more complex for the $2 \mathrm{D} / n \mathrm{D}$ case and again the absence of a single concept of primeness is at the core of the issue and no definitive answer is known. Research in this area continues using, e.g., elementary operations, for background see Galkowski (2001) and other approaches. Results in this area also include those in Dubi (2009); Yan, Xu, Zhao, and Tian (2014).

Positive systems has been the subject of considerable analysis in the control systems literature, including the 2D Fornasini-Marchesini model. The most commonly accepted definition of a positive system is where the input, state and output variables take positive, or at least nonnegative, values. In the case of the state-space model (2) positvity means that the entries in $x, u$ and $y$ take nonnegative values as do the entries in the matrices and the vectors defining the boundary conditions. One motivation for this analysis is that some physical systems, see Fornasini and Valcher (2005), all internal variables are intrinsically nonnegative as they represent concentrations, 
pressures, number of vehicles and so on. Those that can be described by a quarter plane causal 2D model include river pollution modeling, which was an early proposed application for the 2D Fornasini-Marchesini model Fornasini (1991).

In common with the $1 \mathrm{D}$ linear systems case, positivity of the input sequence is a strong constraint in the sense that, e.g., it could prevent local/global reachability of nonnegative states that could be reached by using an unconstrained input sequence, i.e., allowing nonnegative entries. For these systems, structural properties have a combinatorial nature and this has meant that a graph theoretic approach to the analysis of structural properties as in Fornasini and Valcher (2005). Positive 2D systems remain a topic of interest in the research community, see, e.g., Kaczorek (2001) and the literature since the publication of this research text.

Research has also been reported on finite region stability and stabilization of $2 \mathrm{D}$ linear systems, where for systems described by (2), a finite region is defined, for a finite positive integer $N$, as $\{(i, j): i+j \leq N$, and $N$. Recent results include Zhang, Trentelman, Wamg, and Gao (2017). This formulation includes iterative learning control where the trial length is finite and in applications only a finite number of trials will ever be completed. However, in a repetitive process the pass length is finite by definition and the property of strong practical stability Paszke, Dabkowski, Rogers, and Galkowski (2015) has been used in design for applications.

Fault detection and isolation is an important and well researched topic in 1D systems theory and there has been research reported on the same problem for $2 \mathrm{D} / n \mathrm{D}$ linear systems. In particular, there has been work on the 3D Fornasini-Marchesini state-space model with two spatial and one temporal indeterminate. A motivating factor for this research is that such models can be used to represent the dynamics of certain classes of distributed grid sensor networks Sumanasena and Bauer (2011). The 3D structure of this problem requires two spatial independent variables for the plane of operation and one temporal. Moreover, a 4D model is required if the network is operating in 3D space.

Applications such as those in the agricultural and environmental monitoring sectors and, in particular, contaminant propagation detection and structural health monitoring are suitable for grid or mesh topologies and this has, in turn, led to the grid sensor approach. To be of use, such networks must have high reliability and using distributed schemes for signal/information processing in these networks has immediate and signicant benets in terms energy consumption and data throughput. Distributed algorithms also support applications where local actuation is required in response to local detection and hence minimum response delays when compared with a centralized approach.

Among the many techniques for fault detection and isolation for 1D linear systems is the use of geometric control theory, see, e.g., Massoumnia (1986), where several dierent formulations of this general problem were considered. The general problem is to dene functions, known as residuals, such that they are close to zero when no fault is present and their directional properties give information on the presence of a fault when failure has occurred. The geometric approach to fault detection and isolation for the 3D Fornasini-Marchesini state-space model with two spatial and one temporal indeterminate has been considered in Maleki, Rapisarda, Ntogramatzidis, and Rogers (2015) as discussed briey next.

Let $x(i, j, q) \in \mathbb{R}^{n}$ be the local state vector, $y(i, j, q) \in \mathbb{R}^{m}$ the output vector and $u(i, j, q) \in \mathbb{R}^{l}$ the input vector of the system state-space model. Following the notation 
used in Maleki et al. (2015), introduce the shift operating on a vector, say $w$, as

$$
\begin{aligned}
& \left(\sigma_{1} w\right)(i, j, q)=w(i+1, j, q) \\
& \left(\sigma_{2} w\right)(i, j, q)=w(i, j+1, q) \\
& \left(\sigma_{3} w\right)(i, j, q)=w(i, j, q+1) .
\end{aligned}
$$

Then the 3D Fornasini-Marchesini state-space model has the form

$$
\begin{aligned}
\sigma_{1} \sigma_{2} \sigma_{3} x & =A_{1} \sigma_{2} \sigma_{3} x+A_{2} \sigma_{1} \sigma_{3} x+A_{3} \sigma_{1} \sigma_{2} x \\
& +B_{1} \sigma_{2} \sigma_{3} u+B_{2} \sigma_{1} \sigma_{3} u+B_{3} \sigma_{1} \sigma_{2} u \\
y & =C x
\end{aligned}
$$

and is assumed to describe the nominal, or fault-free, system. To model the system dynamics after a sensor or actuator failure has occurred the nominal model is augmented with additional terms to represent the failure mode, i.e.,

$$
\begin{aligned}
\sigma_{1} \sigma_{2} \sigma_{3} x & =A_{1} \sigma_{2} \sigma_{3} x+A_{2} \sigma_{1} \sigma_{3} x+A_{3} \sigma_{1} \sigma_{2} x \\
& +B_{1} \sigma_{2} \sigma_{3} u+\left[\begin{array}{lll}
L_{1}^{1} & \ldots & L_{1}^{k_{1}}
\end{array}\right] \sigma_{2} \sigma_{3}\left[\begin{array}{c}
m_{1}^{1} \\
\vdots \\
m_{1}^{k_{1}}
\end{array}\right] \\
& +B_{2} \sigma_{1} \sigma_{3} u+\left[\begin{array}{lll}
L_{2}^{1} & \ldots & L_{2}^{k_{2}}
\end{array}\right] \sigma_{1} \sigma_{3}\left[\begin{array}{c}
m_{2}^{1} \\
\vdots \\
m_{2}^{k_{2}}
\end{array}\right] \\
& +B_{3} \sigma_{1} \sigma_{2} u++\left[\begin{array}{lll}
L_{3}^{1} & \ldots & L_{3}^{k_{3}}
\end{array}\right] \sigma_{1} \sigma_{2}\left[\begin{array}{c}
m_{3}^{1} \\
\vdots \\
m_{3}^{k_{3}}
\end{array}\right] \\
y & =C x+\left[\begin{array}{lll}
J^{1} & \ldots & J^{p^{\prime}}
\end{array}\right]\left[\begin{array}{c}
n_{1} \\
\vdots \\
n^{p^{\prime}}
\end{array}\right],
\end{aligned}
$$

where (dropping the superscripts and subscripts for ease of notation) the scalars $m, n, p$ and the matrices $L$ and $J$ are termed, respectively, the actuator- and sensor signatures. In Maleki et al. (2015) this model structure is used to design an asymptotic observer (see also the next section on observers for the control of 2D systems described by the Fornasini-Marchesini state-space model) for the nominal (95) and the failure model (96) that takes as inputs the input and output systems variables and produces as the output a residual that asymptotically provides information about the presence and location of the failure. This analysis is in the geometric setting and further development would be required for application to an example. Another setting for fault detection of these systems is to uses polynomial-algebraic tools, see, e.g., Bisisacco and Valcher (2006). 


\section{Controller Design}

Controller design for 2D linear systems has seen a very large volume of research using various representations and settings for analysis. In this section, the structure of the control laws used is first considered and then their design is considered.

A state feedback law for a system described by (5) (discarding the output equation) has the form

$$
u(i, j)=K z(i, j),
$$

resulting in the controlled dynamics state-space model

$$
x(i+1, j+1)=\left(A_{1}+B_{1} K\right) x(i+1, j)+\left(A_{2}+B_{2} K\right) x(i, j+1) .
$$

The design of $K$ for stabilization of the controlled dynamics has been a common topic since after the Fornasini Marchesini model was published. Early contributions can be fund in, e.g., Kaczorek (1985); Lu and Lee (1983). A significant part of this work is for single-input single-output examples.

A significant volume of research on the he design of state feedback control for this case started from (34) where the conditions due to Huang (1972) enables the 2D design problem to be formulated in 1D linear systems terms. The design of a control law of the form (97) is still a subject of fundamental importance, see, eg., Bachelier, Chuzeau, Davod, and Yeganefar (2017) but with the emphasis on stabilization as opposed to stabilization and control.

The results referred to above seek necessary and sufficient conditions for stabilizability but much progress is also possible via the 2D Lyapunov equation, resulting in LMI based designs. If all entries in the state vector are not available then one option is to use an observer to reconstruct the state vector. Moreover, the design of an observer for a 2D linear system follows the same general approach as for 1D linear systems, i.e.,use input/output data to reconstruct the state vector and early work on problem includes Bisiacco (1985a, 1986).

Consider state-space model (3) and let $\hat{x}$ denote the observer state vector. Then the system

$$
\begin{aligned}
\hat{x}(i+1, j+1) & =F_{1} \hat{x}(i+1, j)+\hat{F}_{2} \hat{x}(i, j+1)+G_{1}\left[\begin{array}{l}
u(i+1, j) \\
y(i+1, j)
\end{array}\right] \\
& +G_{2}\left[\begin{array}{l}
u(i, j+1) \\
y(i, j+1)
\end{array}\right] \\
\hat{y}(i, j) & =H \hat{x}(i, j)+J\left[\begin{array}{l}
u(i, j) \\
y(i, j)
\end{array}\right]
\end{aligned}
$$

is said to be Bisiacco (1985a) an exact observer for (3) if: i) it has the finite memory property detailed below ii) and the estimation error

$$
e(i, j)=x(i, j)-\hat{y}(i, j)
$$

vanishes for $i+j$ sufficiently large for all initial conditions.

A system described by (3) is said to have finite memory if the free state, i.e., no input, evolution vanishes for all state initial conditions and $i+j$ sufficiently large. 
Moreover, the finite memory property is equivalent to

$$
\operatorname{det}\left(I-z_{1} A_{1}-z_{2} A_{2}\right)=1 .
$$

A synthesis procedure for an exact observer is also given in Bisiacco (1985a) based on the requirement that (3) is causally reconstructible, i.e., the possibility of computing the state using past values of the inputs and outputs. where for 1D linear systems this property is impled by observability. One necessary and sufficient property for this property to hold for systems described by (3) is that there exist polynomial matrices $P\left(z_{1}, z_{2}\right)$ and $Q\left(z_{1}, z_{2}\right)$ that satisfy the Bezout identity

$$
Q\left(z_{1}, z_{2}\right)\left(I-z_{1} A-z_{2} A_{2}\right)+P\left(z_{1}, z_{2}\right) C=I .
$$

Then Theorem 2 in Bisiacco (1985a) gives that a system described by (3) is causally reconstructible if and only if it admits and exact observer of the form (99).

In the $1 \mathrm{D}$ linear systems case, the observer when it exists is a copy of the original system driven by static control laws acting, respectively, on the output and estimation error. For systems described by (3), the corresponding structure is

$$
\begin{aligned}
\hat{x}(i+1, j+1) & =A_{1} \hat{x}(i+1, j)+A_{2} \hat{x}(i, j+1) \\
& +B_{1} u(i+1, j)+B_{2} u(i, j+1) \\
& +L_{1} e(i+1, j)+L_{2} e(i, j+1), \\
e(i, j) & =y(i, j)-D u(i, j)-C \hat{x}(i, j),
\end{aligned}
$$

for some choice of the constant matrices $L_{1}$ and $L_{2}$. The dynamics of $e(i, j)$ are given by

$$
e(i+1, j+1)=\left(A_{1}-L_{1} C\right) e(i+1, j)+\left(A_{2}-L_{2} C\right) e(i, j+1)
$$

and an exact observer of the form (103) exists if and only if

$$
\operatorname{det}\left(I-\left(A_{1}-z_{1} L_{1}\right) C-z_{2}\left(A_{2}-L_{2} C\right)\right)=1 .
$$

In 1D linear systems theory the geometric approach has provided a setting for solving, in terms of conditions under which a solution exists, of a number of critical problems, such as disturbance decoupling and almost disturbance decoupling with internal stability, see, e.g., Weiland and Willems (1989); Wonham (1974). The geometric approach has also been applied to 2D linear systems, see, e.g., Ntogramatzidis (2012); Ntogramatzidis and Cantoni (2012) (in addition to the fault detection and isolation analysis discussed in Section 7).

\subsection{Optimal Control}

Linear Quadratic Optimal (LQR) control is a well known method and extensively applied method for computing a stabilizing state feedback control law for 1D linear systems. An obvious question then is: can this approach be extended to 2D systems. This problem has received significant attention the literature, see e.g., Bisiacco (1995); 
Bisiacco and Fornasini (1990); Li and Fladali (1995). Focusing on the state equation of (2), a quadratic cost function is

$$
\left.J=\sum_{i+j \geq 0} x(i, j)^{T} Q x(i, j)+u^{T}(i, j) R u(i, j)\right),
$$

where $Q \succeq 0$ and $R \succ 0$ are given weighting matrices.

The solution of this problem was extensively investigated in the cited references above in the 1980's and 1990's with significant progress but substantial differences exist with the 1D linear systems case. For example, unlike the 1D case, it is not always possible to write the 2D linear quadratic optimal control in terms of state feedback. Moreover, differences exist between the Roesser and Fornasini-Marchesini model cases. Also (106) is the infinite frame case and there are again substantial differences in the finite frame case, see, e.g. Ntogramatzidis and Cantoni (2009) (for the Roesser model). Also there has been work on stochastic optimal control for 2D linear systems, including Sebek and Kraus (1995) for the Fornasini-Marchesini model.

More recent work on state feedback control of Fornasini-Marchesini models includesBachelier, Chuzeau, et al. (2017) where the behavorial systems setting is used to develop an approach to stabilization based on equivalence transforms and the property of structural stability. In this case, however, the resulting controlled dynamics are described by a state-space model that is implicit input-free but the corresponding Roesser model is of the standard form. In applications terms, this is an obvious drawback.

An alternative approach to control law design for 2D linear systems is based on LMIs, which leads on to formulas for calculating the stabilizing control law matrix. The potential drawback is, however, that the stability condition is sucient but not necessary and hence the prospect of conservativeness in the design. In particular, an LMI based design could fail to produce a stabilizing control law when such a law exists for a given example.

Despite this limitation, the LMI setting has been used to great effect in the design of control laws for linear repetitive processes and also iterative learning control laws designed using this setting have been experimentally validated, see, e.g., Hladowski et al. (2010, 2012). Moreover, an extension to the case of uncertainty in the system dynamics is straightforward as discussed further below. The LMI approach to stabilization of Roesser and Fornasini Marchesini models has been the subject of many research publication in recent years but the starting point in most cases is based on the 2D Lyapunov equation. On example is the following, which is Theorem 2 in Galkowski et al. (2002).

Theorem 8.1. A $2 D$ discrete linear system described by (3) is stable if there exist matrices $P \succ 0$ and $Q \succ 0$ such that the following LMI is feasible

$$
\left[\begin{array}{cc}
A_{1}^{T} P A_{1}+Q-P & A_{1}^{T} P A_{2} \\
A_{2}^{T} P A_{1} & A_{N}^{T} P A_{2}-Q
\end{array}\right] \prec 0 .
$$

The link to the 2D Lyapunov equation is immediate from this result.

Another approach to stabilizing corol law design is to formulate the problem as a condition that ensures that the controlled dynamics have a particular property. The positive realness property plays an important role in $1 \mathrm{D}$ control and systems theory and applications, e.g., stability analysis for linear systems and the analysis of electrical circuits. In the 2D linear systems case a suitable definition of this property results in 
a stabilizing state feedback control law design with an extension to robust control.

Positive realness for 2D Fornasini-Marchesini systems Xu, Lam, Liu, and Galkowski (2002) is defined as follows.

Definition 8.2. A 2D discrete linear system described by the Fornasini-Marchesini state-space model (2) is said to be:

(1) positive real (PR) if its transfer-function matrix $G\left(z_{1}, z_{2}\right)$ is analytic in $\left|z_{1}\right|>$ $1,\left|z_{2}\right|>1$ and satisfies $G\left(z_{1}, z_{2}\right)+G^{*}\left(z_{1}, z_{2}\right) \succeq 0$ for all $\left|z_{1}\right|>1,\left|z_{2}\right|>1$.

(2) strictly positive real (SPR) if its transfer-function matrix $G\left(z_{1}, z_{2}\right)$ is analytic in $\left|z_{1}\right|>1,\left|z_{2}\right|>1$ and satisfies $G\left(e^{j \theta_{1}}, e^{j \theta_{2}}\right)+G^{*}\left(e^{\theta_{1}}, e^{j \theta_{2}}\right) \succ 0$ for all $\theta_{1}, \theta_{2} \in$ $[0,2 \pi)$.

(3) extended positive real (ESPR) if it is SPR and $G(\infty, \infty)+G^{T}(\infty, \infty) \succ 0$.

The following results are established in Xu et al. (2002).

Theorem 8.3. A $2 D$ discrete linear system described by (2) is asymptotically stable and has the ESPR property if there exist matrices $P \succ 0, Q \succ 0$ and $W \succ 0$ the following LMI is feasible.

$$
\left[\begin{array}{cccc}
A_{1}^{T} P A_{1}+Q-P & A_{1}^{T} P A_{2} & C^{T}-A_{1}^{T} P B_{1} & -A_{1}^{T} P B_{2} \\
A_{2}^{T} P A_{1} & A_{2}^{T} P A_{2}-Q & -A_{2}^{T} P B_{1} & -A_{2}^{T} P B_{2} \\
C-B_{1}^{T} P A_{1} & -B_{1}^{T} P A_{2} & -\left(D+D^{T}-B_{1}^{T} P B_{1}-W\right) & B_{1}^{T} P B_{2} \\
-B_{2}^{T} P A_{1} & -B_{2}^{T} P A_{2} & B_{2}^{T} P B_{1} & B_{2}^{T} P B_{2}-W
\end{array}\right] \prec 0 .
$$

The analysis extends to examples with norm bounded uncertainty as defined by (14)(17).

Theorem 8.4. A $2 D$ discrete linear system described by (14)-(17) is asymptotically stable and has the ESPR property if there exists a scalar $\epsilon>0$ and matrices $X \succ$ $0, Q Y \succ 0$ and $W \succ 0$ the following LMI is feasible.

$$
\left[\begin{array}{cccccc}
Y-X & 0 & X C^{T} & 0 & X A_{1}^{T} & X N_{A 1}^{T} \\
0 & -Y & 0 & 0 & X A_{2}^{T} & X N_{A 2}^{T} \\
C X & 0 & -\left(D+D^{T}-W\right) & 0 & -B_{1}^{T} & -N_{B 1}^{T} \\
0 & 0 & 0 & -W & -B_{2}^{T} & -N_{B 2}^{T} \\
A_{1} X & A_{2} X & -B_{1} & -B_{2} & \epsilon M M^{T}-X & 0 \\
N_{A 1} X & N_{A 2} X & -N_{B 1} & -N_{B 2} & 0 & -\epsilon I
\end{array}\right] \prec 0 .
$$

The following result, stated for the robust control case, shows that the SBR property can give a state stabilizing control law.

Theorem 8.5. Suppose that a control law of the form (97) is applied to a system described by (14)-(17). Suppose also that there exists a scalar $\epsilon>0$ and matrices $X \succ 0, Y \succ 0, W \succ 0$ and $Z$ such that

$$
\left[\begin{array}{ccc}
H & H_{1}^{T} & H_{2}^{T} \\
H_{1} & \epsilon M M^{T}-X & 0 \\
H_{2} & 0 & -\epsilon I
\end{array}\right] \prec 0,
$$


where

$$
\begin{aligned}
H & =\left[\begin{array}{cccc}
Y-X & 0 & X C^{T} & 0 \\
0 & -Y & 0 & 0 \\
C X & 0 & -\left(D+D^{T}-W\right) & 0 \\
0 & 0 & 0 & -W
\end{array}\right], \\
H_{1} & =\left[\begin{array}{cccc}
A_{1} X+N_{B 1 u} Z & A_{2} X+B_{B 2 u} Z & -B_{1} & -B_{2}
\end{array}\right], \\
H_{2} & =\left[\begin{array}{llll}
N_{A 1} X+N_{B 1 u} Z & N_{A 2} X+N_{B 2 u} Z & -N_{B 1} & -N_{B 2}
\end{array}\right],
\end{aligned}
$$

then the controlled dynamics are asymptotically stable and the EPSR property holds. Also a stabilizing control law matrix is

$$
K=Z X^{-1} x(i, j) .
$$

\subsection{Output Feedback Control}

If a stabilizing state feedback control law exists for a given example, it can only be directly implemented if all entries in the state vector are available for measurement. One alternative is to use an observer to reconstruct the state vector (or only those entries that cannot be directly measured) as discussed previously in this section. An alternative is to use static output feedback or a dynamic controller.

A static output feedback control law for (2) replaces the state vector by the output vector, i.e., $u(i, j)=H y(i, j)$. The design of this form of control law has received much attention in the literature together with the links to state feedback control and other systems theoretic properties, see, e.g., Bisiacco (1985b) for substantial early work on the latter aspect and for static output feedback design see, as one of many contributions, Feng, Xu, Wu, and She (2011). Static output feedback control of discrete linear repetitive processes has also been considered, see, e.g., Sulikowski, Galkowski, Rogers, and Owens (2004).

If static control action is insufficient then a dynamic controller is required. For a system described by (2), a dynamic controller has the structure

$$
\begin{aligned}
x^{c}(i+1, j+1) & =A_{1}^{c} x(i+1, j)+A_{2}^{c} x(i, j+1)+B_{1}^{c} y(i+1, j)+B_{2}^{c} y(i, j+1) \\
w(i, j) & =C^{c} x(i, j)+D^{c} y(i, j) .
\end{aligned}
$$

Also, see Bisiacco (1985b), this dynamic controller corresponds to the assumption that the input $u(i, j)$ satisfies a $2 \mathrm{D}$ recursive equation of the form

$$
\begin{aligned}
w(p, q) & =\sum_{\substack{i, j \geq 0 \\
i+j>0}} H_{i, j} u(p-i, q-j) \\
& +\sum_{\substack{i, j \geq 0 \\
i+j>0}} K_{i, j} u(p-i, q-j)+D y(i, j) .
\end{aligned}
$$

Significant progress on the design of this form of controller has been reported with recent results including Chesi and Middleton (2014) (for mixed differential-discrete systems) and Hladowski, Galkowski, Nowicka, and Rogers (2016) in the iterative learning 
control application area with experimental verification. Very recent results in this area are in Chesi and Middleton (2018) (again for the mixed case).

\section{3. $\mathcal{H}_{\infty}$ and $\mathcal{H}_{2}$ Control}

As in $1 \mathrm{D}$ linear systems, the $\mathcal{H}_{\infty}$ and $\mathcal{H}_{2}$ settings have been extensively researched for 2D linear systems. Early work in this area included Du and Xie (2002) and two major areas covered are disturbance rejection and robust control. These are considered in turn next.

Consider the state-space model

$$
\begin{aligned}
x(i+1, j+1) & =A_{1} x(i+1, j)+A_{2} x(i, j+1)+B_{11} w(i+1, j)+B_{12} w(i, j+1), \\
y(i, j) & =C x(i, j)+E w(i, j),
\end{aligned}
$$

with boundary conditions of the form of those for $(2)$ and $w(i, j)$, which is a $2 \mathrm{D}$ exogenous signal representing disturbances. The $2 \mathrm{D}$ transfer-function matrix $G_{d}\left(z_{1}, z_{2}\right)$ relating $Y_{d}\left(z_{1}, z_{2}\right)$ to $W\left(z_{1}, z_{2}\right)$ is

$$
G_{d}\left(z_{1}, z_{2}\right)=C\left(z_{1} z_{2} I-z_{1} A_{1}-z_{2} A_{2}\right)^{-1}\left(z_{1} B_{11}+z_{2} B_{12}\right)+E
$$

and the $\mathcal{H}_{\infty}$ norm of this transfer-function matrix for an asymptotically stable example is

$$
\left\|G_{d}\left(z_{1}, z_{2}\right)\right\|_{\infty}=\sup _{\omega_{1}, \omega_{2} \in[0,2 \pi]} \sigma_{\max }\left(G_{d}\left(e^{j \omega_{1}}, e^{j \omega_{2}}\right)\right)
$$

where $\sigma_{\max }(\cdot)$ denotes the maximum singular value of its matrix argument.

Under zero boundary conditions, an example described by (114) is said to have $\mathcal{H}_{\infty}$ disturbance attenuation $\gamma$ if it is asymptotically stable and its $\ell_{2}$ induced norm is bounded by this scalar, i.e.,

$$
\sup _{0 \neq w \in \ell_{2}} \frac{\left\|y_{d}\right\|_{2}}{\|w\|_{2}}<\gamma \Leftrightarrow\left\|G_{d}\left(z_{1}, z_{2}\right)\right\|_{\infty}<\gamma
$$

where the $\ell_{2}$ norm of $w(i, j)$ is

$$
\|w\|_{2}=\sqrt{\sum_{i=0}^{\infty} \sum_{j=0}^{\infty}\left(\|w(i+1, j)\|^{2}+\|w(i, j+1)\|^{2}\right.} .
$$

Many LMI based results for this property are known. One of them from Du and Xie (2002) is that an example has $\mathcal{H}_{\infty}$ norm bound $\gamma$ if there exist $P \succ 0$ and $Q \succ 0$ such that

$$
\left[\begin{array}{cc}
A^{T} P A-\left[\begin{array}{cc}
P-Q & 0 \\
0 & Q
\end{array}\right]+C^{T} C & C^{T} E+A^{T} P B \\
B^{T} P A+E^{T} C & E^{T} E+B^{T} P B-\gamma^{2} I
\end{array}\right] \prec 0
$$

with $A=\left[\begin{array}{ll}A_{1} & A_{2}\end{array}\right], B=\left[\begin{array}{ll}B_{11} & B_{12}\end{array}\right]$. 
This last analysis can be extended to the case when a control law is applied. Consider the Fornasini-Marchesini state-space model

$$
\begin{aligned}
x(i+1, j+1) & =A_{1} x(i+1, j)+A_{2} x(i, j+1)+B_{11} w(i+1, j)+B_{12} w(i, j+1), \\
& +B_{21} u(i+1, j)+B_{22} u(i, j+1), \\
y(i, j) & =C_{1} x(i, j)+D_{11} w(i, j)+D_{12} u(i, j),
\end{aligned}
$$

to which the control law $u(i, j)=K x(i, j)$ is applied, resulting in the controlled dynamics

$$
\begin{aligned}
x(i+1, j+1) & =\left(A_{1}+B_{21} K\right) x(i+1, j)+\left(A_{2}+B_{22} K\right) x(i, j+1) \\
& +B_{11} w(i+1, j)+B_{12} w(i, j+1), \\
y(i, j) & =\left(C_{1}+D_{12} K\right) x(i, j)+D_{11} w(i, j) .
\end{aligned}
$$

Also this system has $\mathcal{H}_{\infty}$ performance bound $\gamma$ if there exist matrices $W \succ 0$ and $Z \succ 0$ such that

$$
\left[\begin{array}{ll}
\mathcal{A} & \mathcal{B} \\
\mathcal{C} & \mathcal{D}
\end{array}\right] \prec 0
$$

where

$$
\begin{aligned}
& \mathcal{A}=\left[\begin{array}{ccccc}
W & A_{1} W+B_{21} N & A_{2} W+B_{22} N & B_{11} & B_{12} \\
W A_{1}^{T}+N^{T} B_{21} & Z-W & 0 & 0 & 0 \\
W A_{2}^{T}+N^{T} B_{22} & 0 & -Z & 0 & 0
\end{array}\right], \\
& \mathcal{B}=\left[\begin{array}{cc}
0 & 0 \\
W C_{1}^{T}+N^{T} D_{12}^{T} & 0 \\
0 & W C_{1}^{T}+N^{T} D_{12}^{T} \\
D_{11}^{T} & 0 \\
0 & D_{11}^{T}
\end{array}\right] \\
& \mathcal{C}=\left[\begin{array}{ccccc}
0 & C_{1} W+D_{22} N & 0 & D_{11} & 0 \\
0 & 0 & C_{1} W+D_{12} N & 0 & D_{11}
\end{array}\right], \\
& \mathcal{D}=\left[\begin{array}{cc}
-I & 0 \\
0 & -I
\end{array}\right] \text {. }
\end{aligned}
$$

If the LMI of (122) holds, a stabilizing $K$ is given by $K=N W^{-1}$.

The analysis also extends to $\mathcal{H}_{\infty}$ design for uncertain systems and to $\mathcal{H}_{2}$ and mixed $\mathcal{H}_{2} / \mathcal{H}_{\infty}$ design, where the results include those in Chesi and Middleton $(2015,2016)$.

\subsection{Control in the Behavioral Setting}

Control in the behavioral setting for 1D systems takes a contrary view to the standard approach where signal flow graphs process inputs and outputs and control action is viewed as imposing new additional laws on the system Willems (1997). Results on the behavioral approach to the control of $n \mathrm{D}$ systems includes those in Rocha and Wood (2001). Again, differences exist in comparison to the 1D systems case. There has as yet been relatively little research on the implementation of behavioral control designs, some early work is in, e.g., Avelli (2009). 


\section{Nonlinear Dynamics}

The stability and control of nonlinear $2 D / n \mathrm{D}$ dynamics has attracted considerable and increasing interest. Previous research reported includes Yeganefar, Yeganefar, Ghamgui, and Moulay (2013) for the Roesser model structure and Kurek (2014) for the Fornasini-Marchesini model structure. For linear systems as discussed in this paper, some results developed for one representation can be applied to the other, e.g., Roesser to Fornasini-Marchesini and vice versa. This is much less likely for nonlinear dynamics and theory coupled with control law design must proceed separately for each model structure.

This section gives results on stability and control law design for systems described by (27) and (28) where a vector Lyapunov function is employed. It is assumed that there exist finite real numbers $\rho>0, \sigma>0$ and $0<\xi_{0}<1$ such that

$$
\|x(i, 0)\|^{2}=\left\|\xi_{0}(i)\right\|^{2} \leq \rho \zeta_{0}^{i}, i \geq 0,\|x(0, j)\|^{2}=\left\|\eta_{0}(j)\right\|^{2} \leq \sigma \zeta_{0}^{j}, j \geq 0,(i, j) \neq(0,0) .
$$

where throughout this section $\|\cdot\|$ denotes the Euclidean norm on vectors. Moreover, $\zeta_{0}$ represents the rate of convergence in $i$ and $j$ of the initial local state vector sequences. From this point onwards, all references to the boundary conditions will assume that they satisfy (123). The nonlinear functions forming the vector-valued function on the right-hand of (27) are assumed to be single-valued and hence for given boundary conditions solutions are uniquely defined step by step by an explicit recursive rule.

The stability theory developed for 2D discrete linear Fornasini-Marchesini systems has been defined in both the internal, or state, and bounded-input bounded-output settings. Previous research on the stability of $2 \mathrm{D}$ discrete nonlinear systems described by this model includes Kurek (2014), where stability and asymptotic stability were defined and sufficient conditions for their existence obtained in a manner similar to the second Lyapunov stability theorem. This analysis did not lead, however, to control law design. Moreover, as in the 1D systems case, it is to be expected that a stronger form of stability will be required in applications.

In previous work Emelianova, Pakshin, Galkowski, and Rogers (2015), the following definition has been used for autonomous systems, i.e., no inputs.

Definition 9.1. An autonomous 2D discrete nonlinear system described by (27) is said to be exponentially stable if for all boundary conditions satisfying (123) there exist $\kappa>0$ and $0<\lambda<1$ such that

$$
\|x(i, j)\|^{2} \leq \kappa \lambda^{i+j}, i, j \geq 0,(i, j) \neq(0,0) .
$$

In the linear dynamics case, the exponential stability property was considered in Pandolfi (1984). In (124) a common $\kappa$ for all boundary conditions, satisfying (123) is considered, where the structure of these boundary conditions ensures that such a choice is always possible. This stability theory is physically motivated by applications where such a system would never be operated with boundary conditions that can diverge as the dynamics evolve. For the autonomous case it requires that the state vector decays to zero as $i+j \rightarrow \infty$ and is a strong form of stability since the boundary conditions are required to have a uniform convergence property.

As in the 1D nonlinear case, a natural route to characterizing exponential stability would be based on a suitably chosen Lyapunov function. This approach is based on 
properties of the function itself and for discrete dynamics of its increments. In the 2D systems case, however, the dynamics are governed by vector valued functions of the independent variables $i$ and $j$. One way would be to chose a candidate Lyapunov function as a scalar function, say $V(i, j)$, with the same properties as the 1D case and then construct the gradient along the system trajectories. However, this last step requires $x(i+1, j)-x(i, j)$ and $x(i, j+1)-x(i, j))$. However, these quantities can only be found by solving (27), but then all of the advantages of the Lyapunov approach are lost.

As an alternative, previous work has used a vector Lyapunov function approach for other classes of 2D nonlinear systems, see, e.g., Emelianova et al. (2015), where for discrete dynamics a counterpart of divergence was used instead of the gradient. In what follows, a similar setting is used to characterize the property of Definition 9.1 using a vector Lyapunov function of the form

$$
V(x(i, j+1), x(i+1, j))=\left[\begin{array}{l}
V_{1}(x(i+1, j+1)) \\
V_{2}(x(i+1, j+1))
\end{array}\right],
$$

where $V_{1}(x) \neq 0, x(i+1, j+1) \neq 0, V_{2}(x) \neq 0, x(i+1, j+1) \neq 0$ and $V_{1}(0)=0$ and $V_{2}(0)=0$. Also the counterpart of the divergence operator, referred to as the gradient from this point onwards, of this function along the trajectories of (27) is

$$
\begin{aligned}
\mathcal{D}(x(i, j+1), x(i, j+1)) & =V_{1}(i+1, j+1)-V_{1}(x(i, j+1)) \\
& +V_{2}\left(x(i+1, j+1)-V_{2}(x(i+1, j)) .\right.
\end{aligned}
$$

The following result is Theorem 2.2 in Pakshin, Emelianova, Galkowski, and Rogers (2018).

Theorem 9.2. An autonomous $2 D$ discrete nonlinear system described by (27) and boundary conditions that satisfy (123) is exponentially stable in the sense of Definition 9.1 if there exists a vector function of the form (125) and positive scalars $c_{1}, c_{2}$ and $c_{3}$ such that

$$
\begin{aligned}
c_{1}\|x(i, j+1)\|^{2} & \leq V_{1}(x(i, j+1)) \leq c_{2}\|x(i, j+1)\|^{2}, \\
c_{2}\|x(i+1, j)\|^{2} & \leq V_{1}(x(i+1, j)) \leq c_{2}\|x(i+1, j)\|^{2}, \\
\mathcal{D} V(x(i, j+1), x(i+1, j)) & \leq-c_{3}\left(\|x(i, j+1)\|^{2}+\|x(i+1, j)\|^{2}\right) .
\end{aligned}
$$

Given this stability theory, the obvious follow on questions is: can this theory form the basis for control law design? One way of addressing this question is considered in Pakshin et al. (2018) based on passivity theory, which is well established in nonlinear 1D systems theory, see, e.g., Fradkov and Hill (1998) and Willems (1972). In recent work Pakshin et al. (2018), a passivity approach to the stabilization of 2D nonlinear systems described by the Fornasini-Marchesini state-space model that also makes use of a vector storage function of the form (125) has been developed. The central results are given next with an application to the case where the dynamics are linear and the nonlinearity enters through the actuator dynamics, e.g., as a result of saturation.

Introduce, for passification based control law design, see also the discussion after the definition, the auxiliary vector $z(i, j) \in \mathbb{R}^{n_{z}}$ given by

$$
z(i, j)=h(\bar{x}(i, j), \bar{u}(i, j)),
$$


where

$$
\begin{aligned}
& \bar{x}(i, j)=\left[\begin{array}{ll}
x^{T}(i, j+1) & x^{T}(i+1, j)
\end{array}\right]^{T}, \\
& \bar{u}(i, j)=\left[\begin{array}{ll}
u^{T}(i, j+1) & u^{T}(i+1, j)
\end{array}\right]^{T} .
\end{aligned}
$$

Also $h$ is a nonlinear function where it is assumed that $h(0,0)=0$ and hence an equilibrium at the origin and the passivity property is defined as follows.

Definition 9.3. A 2D discrete nonlinear system described by (27) with boundary conditions that satisfy (123) is said to be exponentially passive if there exists a vector function (125), a vector $z$ of the form (128) and positive scalars $c_{1}, c_{2}$ and $c_{3}$ satisfying the first two inequalities in (127) and

$$
\mathcal{D} V(x(i, j+1), x(i+1, j)) \leq z^{T}(i, j) G \bar{u}(i, j)-c_{3}\left(\|x(i, j+1)\|^{2}+\|x(i, j+1)\|^{2}\right),
$$

where $G$ is a constant matrix of compatible dimensions.

This last definition is an extension of that for 1D systems, see, e.g., Fradkov and Hill (1998), where the vector $z$ can be regarded as auxiliary output vector that is used to construct a control law such that the controlled process has the passivity property. The selection such a vector is known in the passivity theory for $1 \mathrm{D}$ systems as passivation or passification. Moreover, the choice of this vector depends on the choice of a vector storage function $V$ and it is a separate complex problem (similar to the choice of a Lyapunov function for a nonlinear system). The problem is to find a pair $(z, V)$ satisfying (129), which is then further developed for a particular applications relevant case. The following result is Theorem 3.2 in Pakshin et al. (2018).

Theorem 9.4. Suppose that a $2 D$ discrete nonlinear system described by (27) and (123) is exponentially passive in the sense of Definition 9.3. Suppose also that there exists a function $\psi(z)$ such that $\psi(0)=0$ and $z^{T} G \psi(z)>0$ if $z \neq 0$. Then the control law

$$
\bar{u}(i, j)=-\psi(z(i, j)
$$

results in controlled dynamics that are exponentially stable.

The storage function (125) can be considered as a vector Lyapunov function for the controlled process formed by applying a control law of the form (130) to (27) that guarantees exponential stability. One particular sub-problem is now considered. This is for the case where the dynamics are linear but nonlinearity arises in implementation, with state-space model

$$
\begin{aligned}
x(i+1, j+1) & =A_{1} x(i, j+1)+A_{2} x(i+1, j)+\phi_{1}(x(i, j+1), x(i+1, j)) u(i, j+1) \\
& +\phi_{2}\left(x_{i, j+1}, x_{i+1, j}\right) u_{i+1, j},
\end{aligned}
$$

where $\phi_{1}$ and $\phi_{2}$ are compatibly dimensioned matrices with nonlinear functional entries. One simple but practically relevant case is when $\phi_{1}$ and $\phi_{2}$ are constant matrices and then (131) is a linear Fornasini-Marchesini state-space model. This model is one representation of the applications relevant case where the dynamics are linear, or can 
reasonably be approximated as such and in operation the actuators exhibit nonlinearities such as saturation.

Introduce the notation

$$
A=\left[\begin{array}{ll}
A_{1} & A_{2}
\end{array}\right], \phi(\bar{x}(i, j))=\left[\phi_{1}(x(i, j+1), x(i+1, j)) \phi_{2}(x(i, j+1), x(i+1, j))\right] .
$$

Also choose the storage function for (131) in the form (125) with $V_{1}(x)=$ $\alpha x^{T} P x, V_{2}(x)=\beta x^{T} P x, P \succ 0$ and the scalars $\alpha>0, \beta>0$ such that $\alpha+\beta=1$. Suppose also that $P$ satisfies the Lyapunov-type linear matrix inequality

$$
\left[\begin{array}{cc}
A_{1}^{T} P A_{1}-\alpha P+Q_{11} & A_{1}^{T} P A_{2}+Q_{12} \\
A_{2}^{T} P A_{1}+Q_{12}^{T} & A_{2}^{T} P A_{2}-\beta P+Q_{22}
\end{array}\right] \prec 0,
$$

where $Q=\left[\begin{array}{ll}Q_{11} & Q_{12} \\ Q_{12}^{T} & Q_{22}\end{array}\right] \succ 0$. Define for passification based control law design, an auxiliary output vector for (131) as

$$
z(i, j)=2 \phi^{T}\left(\bar{x}(i, j) P \bar{x}(i, j)+\frac{1}{2} \phi^{T}(\bar{x}(i, j) P \phi(\bar{x}(i, j)) \bar{u}(i, j),\right.
$$

where in this case

$$
\begin{aligned}
\mathcal{D} V(x(i, j+1), x(i+1, j)) & =\bar{x}^{T}(i, j)\left(A^{T} P A-\left[\begin{array}{cc}
\alpha P & 0 \\
0 & \beta P
\end{array}\right]\right) \bar{x}(i, j) \\
& +2 \bar{x}^{T}(i, j) A^{T} P(\bar{x}(i, j)) \bar{u}(i, j) \\
& +\bar{u}^{T}(i, j) \phi^{T}(\bar{x}(i, j)) \bar{u}(i, j) \leq 2 z^{T}(i, j) \bar{u}(i, j) \\
& -\lambda_{\min }(Q)\|\bar{x}\|^{2}
\end{aligned}
$$

and $\lambda_{\min }(Q)>0$ denotes the smallest eigenvalue of $Q$. Then it follows immediately from (134) that the system described by (131)-(133) is exponentially passive with $G=2 I$. Moreover, by Theorem 9.4 the control law

$$
\bar{u}(i, j)=-\left[I+\phi^{T}(\bar{x}(i, j)) P \phi(\bar{x}(i, j))\right]^{-1} \phi^{T}(\bar{x}) P A \bar{x}(i, j),
$$

applied to (131) results in a controlled system that is exponentially stable. Much further development of the basic results in this section is possible and is an area of ongoing research.

\section{Applications}

The substantial progress on the development of a systems theory for 2D linear systems described by the Fornasini-Marchesini model has not led to a similar level of interest in terms of applications. Early on there was the river pollution modeling Fornasini (1991) that established a role for the Fornasini-Marchesini model but with limited follow up. Also there continues to be research conducted on the use of $2 \mathrm{D}$ systems analysis for convolutional codes, see, e.g., Pinto, Pinto, and Rocha (2014), Valcher and Fornasini (1994) (where the second reference uses the Roesser model but links back to the 2D realization problem discussed in Section 7). 
One application area that has seen verification studies is distributed information processing in grid sensor networks, see, e.g., Sumanasena and Bauer (2011), which, in turn, cites relevant $n \mathrm{D}$ systems theory. This research has shown that linear systems can be implemented in a distributed fashion in real-time on a grid sensor network under the condition that the system equations satisfy a particular constrained Fornasini-Marchesini model. Again this relates, in particular, to the realization problem discussed in Section 7 and also the fault detection and isolation work discussed in this same section. An emerging application area for the results of Section 9 is laser model deposition, see, e.g., Sammons, Gegel, Bristow, and Landers (2018), where a linear model is an inadequate representation of the dynamics.

\section{Conclusions}

In the four decades since its publication, the Fornasini-Marchesini state-space model for $2 \mathrm{D} / n \mathrm{D}$ dynamics has seen a substantial body of systems theory developed from a number of starting points. In the control systems area, however, the number of applications where a $2 \mathrm{D}$ systems representation is the only possible starting point awaits a convincing example. This is in contrast to the related areas of signal and image processing. Application areas do exist, however, where the 2D systems theory provides a means of answering systems theoretic/design problems, where the most prominent of these is iterative learning control. For nonlinear dynamics, the laser applications area is an application for suitably developed systems theory/control law design.

\section{Funding}

This work is partially supported by National Science Centre in Poland, grant No. 2015/17/B/ST7/03703.

\section{References}

Agathoklis, P., Jury, E. I., \& Mansour, M. (1993). Algebraic necessary and sufficient conditions for the stability of 2-D discrete systems. IEEE Transacitons on Circuits and Systems II: Analog and Digital Signal Processing, 40(4), 251-258.

Anderson, B. D. O., Agathoklis, P., Jury, E. I., \& Mansour, M. (1986). Stability and the matrix Lyapunov equation for discrete $2 \mathrm{D}$ linear systems. IEEE Transacitons on Circuits and Systems, CAS-33, 261-267.

Attasi, S. (1973). Systemes lineaires homogenes a deux indices (Vol. 31; Tech. Rep.). IRIA.

Avelli, D. N. (2009). Almost direct sum decomposition and implementation of 2D bheaviors. Mathematics of Control, Signals, and Systems, 21, 1-19.

Bachelier, O., Chuzeau, T., Davod, R., \& Yeganefar, N. (2017). Structural stablilization of linear 2D discrete systems using equivalence transformations. Multidimensional Systems and Signal Processing, 28(4), 1629-1652.

Bachelier, O., Paszke, W., Yeganefar, N., Mehdi, D., \& Cherifi, A. (2016). LMI stability conditions for 2D Roesser models. IEEE Transactions on Automatic Control, 61(3), 766770 .

Bachelier, O., Paszke, W., Yeganefar, N., Mehdi, D., \& Cherifi, A. (2017). Towards necessary and sufficient stability conditions for 2D Roesser models. IEEE Transactions on Automatic Control, 61(3), 766-770. 
Barmish, B. R. (1994). New tools for robustness of linear systems. New York: Macmillian.

Bisiacco, M. (1985a). On the state reconstruction of 2D systems. Systems and Control Letters, 5, 347-353.

Bisiacco, M. (1985b). State and output-feedback stabilizability of 2-D systems. IEEE Transactions on Circuits and Systems, CAS-32(12), 1246-1254.

Bisiacco, M. (1986). On the structure of 2-D observers. IEEE Transactions on Automatic Control, 31(7), 676-680.

Bisiacco, M. (1995). New results in 2D optimal control theory. Multidimensional Systsms and Signal Perocessing, 6, 189-222.

Bisiacco, M., \& Fornasini, E. (1990). Optimal control of 2-D systems. SIAM Journal on Control and Optimization, 28, 582-601.

Bisisacco, M., \& Valcher, M. E. (2006). Observer-ased fault detection and isolation of 2-D state-space models. Multidimensional Systems and Signal Processing, 17, 219-242.

Bliman, P.-L. (2002). Lyapunov equation for the stability of 2-D systems. Multidimensional Systems and Signal Processing, 13, 201-222.

Chesi, G., \& Middleton, R. (2014). Necessary and sufficient LMI conditions for stability and performance analysis of 2-D mixed continuous-discrete-time systems. IEEE Transactions on Automatic Control, 59(4), 996-1007.

Chesi, G., \& Middleton, R. (2015). $H_{\infty}$ and $H_{2}$ norms of 2D mixed continuous-discrete-time systems. IEEE Transactions on Automatic Control, 60(10), 2614-2625.

Chesi, G., \& Middleton, R. (2016). Robust stability and performnce analysis of 2Dmixed continuous-discrete-time systems with uncertainty. Automatica, 67, 233-243.

Chesi, G., \& Middleton, R. (2018). LMI based fixed-order output feedback systems for twodimensional mixed continuous-discrete-time systems. IEEE Transactions on Automatic Control, 63(4), 960-972.

Du, C., \& Xie, L. (2002). $H_{\infty}$ control and filtering of two-dimensional systems (Vol. 278). Berlin, Germany: Springer-Verlag.

Dubi, C. (2009). Triangular representation of Fornasini-Marchesini systems. Multidimensional Systems and Signal Processing, 20(3), 217-234.

Dymkov, M., Gaishun, I., Rogers, E., Galkowski, K., \& Owens, D. H. (2003). z-transform and volterra-operator based approaches to controllability and observability analysis for discrete linear repetitive processes. Multidimensional Systems and Signal Processing, 14(4), 365395.

Ebihara, Y., Ito, Y., \& Hagiwara, T. (2006). Exact stability analysis of 2-D systems using LMIs. IEEE Transactions on Automatic Control, 51 (9), 1509-1513.

Emelianova, J., Pakshin, P., Galkowski, K., \& Rogers, E. (2015). Stability of nonliner discrete repetitive processes with Markovian switching. Systems and Control Letters, 75, 108-116.

Feng, Z.-Y., Xu, L., Wu, M., \& She, J.-H. (2011). $H_{\infty}$ static output feedback control of 2-D systems in FM secnd model. Asian Journal of Control, 14(6), 1505-1513.

Fornasini, E. (1991). A 2-D apporach to river pollution modeling. Multidimensional Systems and Signal Processing, 2(3), 233-265.

Fornasini, E., \& Marchesini, G. (1978). Doubly-indexed dynamical systems: state-space models and structural properties. Mathematical Systems Theory, 12, 59-72.

Fornasini, E., \& Marchesini, G. (1980). Stability analysis of 2-D systems. IEEE Transactions on Circuits and Systems, CAS-27(12), 1210-1217.

Fornasini, E., \& Valcher, M. E. (2005). Controllability and reachability of 2-D positive systems: a graph theoretic approach. IEEE Transactions on Circuits and Systems I: Regular Papers, 52(3), 576-585.

Fradkov, A., \& Hill, D. (1998). Exponential feedback passivity and stabilizability of nonlinear systems. Automatica, 34, $697-703$.

Frost, M. G., \& Boudellioua, M. S. (1989). Further observations concerning the strict system equivalence of polynomial system matrices over $\mathbb{R}[s, z]$. International Journal of Control, 49, 1-14.

Fuhrmann, P. A. (1977). On strict system equivalence and similarity. International Journal 
of Control, 25, 5-10.

Galkowski, K. (2001). State-space realization for 2-D linear systems with extensions to the general $n D(n>2)$ case (Vol. 263). Berin, Germany: Springer.

Gałkowski, K., Rogers, E., \& Owens, D. (1995). New 2D models and a transition matrix for discrete linear repetitive processes. Int. J. Control, 72(15), 1365-1380.

Gałkowski, K., Rogers, E., \& Owens, D. H. (1998). Matrix rank based conditions for reachability / controllability of discrete linear repetitive processes. Linear Algebra and its Applications, 275-276, 201-224.

Galkowski, K., Rogers, E., Xu, S., Lam, J., \& Owens, D. H. (2002). LMIs - a fundamental tool in analysis and controller design for discrete linear repetitive processes. IEEE Transactions on Circuits and Systems I, Fundamental Theory and Applications, 49(6), 768-778.

Hinamoto, T. (1993). 2-D lyapunov equation and filter design based on the fornasini-marchesini second model. IEEE Transacitons on Circuits and Systems I: Fundamental Theory and Applications, 40(2), 102-110.

Hladowski, L., Galkowski, K., Cai, Z., Rogers, E., Freeman, C. T., \& Lewin, P. L. (2010). Experimentally supported 2D systems based iterative learning control law design for error convergence and performance. Control Engineering Practice, 18, 339-348.

Hladowski, L., Galkowski, K., Cai, Z., Rogers, E., Freeman, C. T., \& Lewin, P. L. (2012). Output information based iterative learning control law design with experimental verification. ASME Journal of Dynamic Systems, Measurement and Control, 134(2), 021012/1$021012 / 10$

Hladowski, L., Galkowski, K., Nowicka, W., \& Rogers, E. (2016). Repetitive process based design and experimental verification of a dynamic iterative learning control law. Control Engineering Practice, 46, 157-165.

Huang, T. S. (1972). Stability of two-dimensional recursive filters. IEEE Transactiona on Audio Electrocoustics, AU-20, 158-163.

Justice, J. H., \& Shanks, J. L. (1973). Stability criterion for $n$-dimensional digital filters. IEEE Transactions on Automatic Control, AC-18, 284-286.

Kaczorek, T. (1985). Two-dimensional linear systems (Vol. 68). Berlin: Springer.

Kaczorek, T. (2001). Positive $1 D$ and $2 D$ systems (Vol. 68). Berlin: Springer.

Kailath, T. (1980). Linear systems. Englewood Cliffs, NJ: Prentice-Hall.

Kalman, R. E., Falb, P., \& Arbib, M. (1969). Topics in mathematical system theory. Monteral: McGraw-Hill.

Kurek, J. E. (1985). The general state-space model for two-dimensional linear digital systems. IEEE Transactions on Automatic Control, AC-30(6), 600-602.

Kurek, J. E. (2014). Stability of nonlinear time-varying digital 2-D Fornasini-Marchesini systems. Multidimensional Systems and Signal Processing, 25, 600-602.

Lang, S. (1997). Algebra 3rd edition. Addison Wesley.

Li, C., \& Fladali, M. (1995). Optimal control of 2-D systems. Multidimensional Systsms and Signal Perocessing, 6, 189-222.

Lu, W.-S., \& Lee, E. B. (1983). Stability analysiss for two-dimensional systems. IEEE Transactions on Circuits and Systems, CAS-30(7), 455-461.

Maleki, S., Rapisarda, P., Ntogramatzidis, L., \& Rogers, E. (2015). Failure identification for 3D linear systems. Multidimensional Systems and Signal Processing, 26, 481-502.

Malgrange, B. (1962/63). Systemes differentiels a coefficients constants. Seminaire Bourbaki, $1-11$.

Massoumnia, M. A. (1986). A geometric approach to the synthesis of failure detection filters. IEEE Transactions on Automatic Control, 31, 839-849.

Ntogramatzidis, L. (2012). Structural invariants of two-dimensional systems. SIAM Journal on Control and Optimization, 50(1), 334-356.

Ntogramatzidis, L., \& Cantoni, M. (2009). LQ optimal control for 2-D Roesser models of finite extent. Systems and Control Letters, 58, 482-490.

Ntogramatzidis, L., \& Cantoni, M. (2012). Structural invariants of two-dimensional systems. SIAM Journal on Control and Optimization, 50(1), 334-356. 
Oberst, O. (1990). Multidimensional constant linear systems. Acta Applicandae Mathematicae, 20, $1-175$.

Oberst, O. (1995). Variations on the fundamental principle for linear systems of partial differential and difference equations with constant coefficients. Applicable Algebra in Engineering Communication and Computing, 6(4-5), 221-243.

Ooba, T. (2000). On stability analysis of 2-D systems on 2-D Lyapunov matrix inequalities. IEEE Transactions on Circuits and Systems I, Fundamental Theory and Applications, $47(8), 1263-1265$.

Owens, D. H., \& Rogers, E. (1995). Frequency domain Lyapunov equations and performance bounds for differential linear repetitive processes. Systems and Control Letters, 26(1), 6568.

Pakshin, P., Emelianova, J., Galkowski, K., \& Rogers, E. (2018). Stabilization of 2D nonlinear systems described by Fornasini-Marchesini models. SIAM Journal on Control and Optimization, accepted.

Pandolfi, L. (1984). Exponential stability of 2D systems. Systems and Control Letters, 4, 381-385.

Paszke, W., Dabkowski, P., Rogers, E., \& Galkowski, K. (2015). New results on strong practical stability and stabilization of discrete linear repetitive processes. Systems $\&$ Control Letters, 77, 22-29.

Paszke, W., Rogers, E., \& Galkowski, K. (2016). Experimentally verified generalized KYP lemma based iterative learning control design. Control Engineering Practice, 53, 57-67.

Pinto, T., Pinto, R., \& Rocha, P. (2014). Realization of 2D convolutional codes of rate $\frac{1}{n}$ by separable Roesser models. Desing, Codes and Cryptography, 70(1), 241-250.

Polderman, J. W., \& Willems, J. C. (1998). Introduction to mathematical systems theory a behavioral approach (Vol. 26). Berlin: Springer.

Pugh, A. C., McInerney, S. J., Boudellioua, M. S., Johnston, D. S., \& Hayton, G. E. (1998). A transformation for 2-D linear systems and a generalization of a theorem of Rosenbrock. International Journal of Control, 71 (3), 491-503.

Rocha, P. (1990). Structure and representation of 2-D systems (Unpublished doctoral dissertation). University of Groningen, The Netherlands.

Rocha, P., \& Willems, J. C. (1989). State for 2D systems. Linear Algebra and its Applications, 122/123/124, 1001-1038.

Rocha, P., \& Willems, J. C. (1991). Controllability of 2-D systems. IEEE Transactions on Automatic Control, 36(4), 413-423.

Rocha, P., \& Wood, J. (2001). Trajectory control and interconnection of 1D and 2D systems. SIAM Journal on Control and Optimization, 40(1), 107-134.

Roesser, R. P. (1975). A discrete state-space model for linear image processing. IEEE Transactions on Automatic Control, 20(1), 1-10.

Rogers, E., Galkowski, K., \& Owens, D. H. (2007). Control systems theory and applications for linear repetitive processes (Vol. 349). Berlin: Springer, Lecutre Notes in Control and Information Sciences.

Rogers, E., \& Owens, D. H. (1993). Stability tests and performance bounds for a class of 2D linear systems. Multidimensional Systems and Signal Processing, 4, 355-391.

Rosenbrock, H. H. (1970). State-space and multivariable theory. London: Nelson.

Sammons, P. M., Gegel, M. L., Bristow, D. A., \& Landers, R. G. (2018). Repetitive process control of additive manufacturing with application to laser metal deposition. IEEE Transactions on Control Systems Technology, DOI: 10.1109/TCST.2017.2781653.

Sebek, M., \& Kraus, F. J. (1995). Stochastic LQ control for 2-D systems. Multidimensional Systems and Signal Processing, 6, 275-285.

Sulikowski, B., Galkowski, K., Rogers, E., \& Owens, D. H. (2004). Output feedback control of discrete linear repetitive processes. Automatica, 40, 2167-2173.

Sumanasena, M. G. B., \& Bauer, P. H. (2011). Realizations using the Fornasini-Marchesini model for implememntation in distributed grid sensor networks. IEEE Transactions on Circuits and Systems I: Regular Papers, 58(1), 2708-2717. 
Valcher, M. E. (2000). Characteristic cones and stability properties of two-dimensional autonomous behaviors. IEEE Transactions on Circuits and Systems I: Fundamental Theory and Applications, 47(3), 290-302.

Valcher, M. E., \& Fornasini, E. (1994). On 2D finite support convolutional codes: an algebraic approach. Multidimensional Systems and Signal Processing, 5, 231-243.

Weiland, S., \& Willems, J. C. (1989). Almost disturbance decoupling with internal stability. IEEE Transactions on Automatic Control, 34(3), 277-286.

Willems, J. C. (1972). Dissipative dynamical systems part I: General theory. Archives for Rational Mechanics and Analysis, 45, 325-351.

Willems, J. C. (1991). Paradigms and puzzles in the theory of dynamical systems. IEEE Transactions on Automatic Control, 36, 259-294.

Willems, J. C. (1997). On interconnections, control, and feedback. IEEE Transactions on Automatic Control, 42(3), 326-339.

Wonham, W. M. (1974). Linear multivariable control: A geometric approach. New York: Springer.

Wood, J., Oberst, U., Rogers, E., \& Owens, D. H. (2000). A behavioral approach to the pole structure of one-dimensional and multidimensional linear systems. SIAM Journal of Control and Optimization, 38(2), 627-661.

Wood, J., Rogers, E., \& Owens, D. H. (1999). Controllable and autonomous $n$ d linear systems. Multidimensional Systems and Signal Processsing, 10, 33-69.

Xu, S., Lam, J., Liu, Z., \& Galkowski, K. (2002). Positive real control for uncertain twodimensional systems. IEEE Transactions on Circuits and Systems I: Regular Papers, 49(11), 1659-1666.

Yan, S., Xu, L., Zhao, Q., \& Tian, Y. (2014). Elementary operation approach to order reduction for Roesser state-space model of multidimensional systems. IEEE Transactions on Circuits and Systems I: Regular Papers, 61(3), 789-802.

Yeganefar, N., Yeganefar, N., Ghamgui, M., \& Moulay, E. (2013). Lyapunov theory for 2D nonlinear Roesser models: Application to asymptotic and exponential stability. IEEE Transactions on Automatic Control, 58, 1299-1304.

Youla, D. C., \& Gnavi, G. (1979). Notes on $n$-dimensional system theory. IEEE Transactions on Circuits and Systems, CAS-26, 105-111.

Zaris, P. M., Wood, J., \& Rogers, E. (2001). Controllable and uncontrollable poles and zeros of $n$ d linear systems. Mathematics of Control Signals and Systems, 14, 281-298.

Zerz, E. (2000a). On strict system equivalence for multidimensional systems. International Journal of Control, $73(6), 495-504$.

Zerz, E. (2000b). Topics in multidimensional linear systems theory (Vol. 256). London, England: Springer-Verlag.

Zhang, G., Trentelman, H. L., Wamg, W., \& Gao, J. (2017). Input-output finite-region stability and stabilization for discrete 2-D Fornasini-Marchesini models. Systems 8 Control Letters, 99, 9-16. 